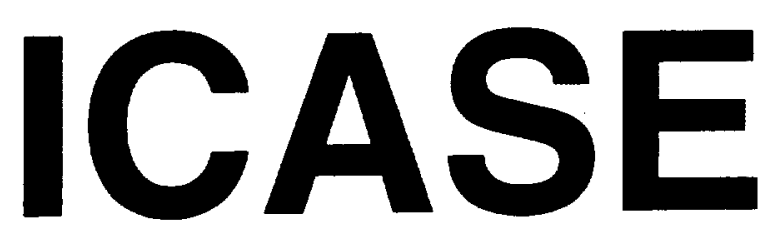

\title{
SPLINE APPROXIMATION OF THIN SHELL DYNAMICS
}

\section{R. C. H. del Rosario \\ R. C. Smith}

NASA Contract No. NASI-19480

March 1996

Institute for Computer Applications in Science and Engineering NASA Langley Research Center Hampton, VA 23681-0001

Operated by Universities Space Research Association

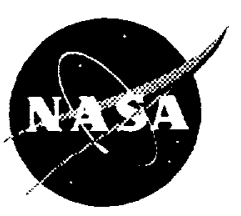

National Aeronautics and Space Administration

Langley Research Center

Hampton, Virginia 23681-0001 



\title{
SPLINE APPROXIMATION OF THIN SHELL DYNAMICS ${ }^{1}$
}

\author{
R.C.H. del Rosario \\ Center for Research in Scientific Computation \\ North Carolina State University \\ Raleigh, NC 26795 \\ R.C. Smith \\ Department of Mathematics \\ Iowa State University \\ Ames, IA 50011
}

\begin{abstract}
A spline-based method for approximating thin shell dynamics is presented here. While the method is developed in the context of the Donnell-Mushtari thin shell equations, it can be easily extended to the Byrne-Flügge-Lur'ye equations or other models for shells of revolution as warranted by applications. The primary requirements for the method include accuracy, flexibility and efficiency in smart material applications. To accomplish this, the method was designed to be flexible with regard to boundary conditions, material nonhomogeneities due to sensors and actuators, and inputs from smart material actuators such as piezoceramic patches. The accuracy of the method was also of primary concern, both to guarantee full resolution of structural dynamics and to facilitate the development of PDE-based controllers which ultimately require real-time implementation. Several numerical examples provide initial evidence demonstrating the efficacy of the method.
\end{abstract}

\footnotetext{
${ }^{1}$ This research was supported in part by the National Aeronautics and Space Administration under NASA Contract Number NAS1-19480 while the authors were visiting scientists at the Institute for Computer Applications in Science and Engineering (ICASE), NASA Langley Research Center, Hampton, VA 23681. Additional support was also provided in part under NASA grant NAG-1-1600.
} 



\section{Introduction}

A spline-based method for numerically approximating thin shell dynamics is presented in this paper. This research is motivated by the growing number of applications involving shell models for which explicit solutions are typically unavailable. A specific example involves the development of control strategies for reducing vibrations, fatigue and structure-borne noise in aircraft fuselages. Typically, some form of cylindrical shell model is employed with the specific equations and boundary conditions dictated by the application or experimental setup. When numerically discretizing the models in such applications, the approximation method must be flexible with respect to boundary conditions and adaptable with regard to nonhomogeneities in materials and geometries. For example, if piezoceramic patches bonded to or embedded in the shell are used as actuators or sensors, the numerical method must be sufficiently flexible so as to permit extension to models incorporating these components. Also, the primary motion in many vibration and noise control applications is bending-dominated and the numerical method must accurately approximate such dynamics. Finally, from a control perspective, the approximation method must adequately preserve stability properties of the physical system as well as be sufficiently efficient so as to permit real-time implementation.

Current techniques for approximating shell dynamics include modal expansions [17, 18, 26], finite element discretizations $[2,3,4,11,13,14,15,20,21,22,25,28,30]$ and finite difference approximations [31,32]. From a theoretical perspective, modal expansions arise naturally when separating variables in linear shell models. In models for which analytic expressions for the eigenfunctions or modes can be obtained, this provides a straightforward and often quite efficient method of approximation. The difficulty, however, lies in the fact that analytic expressions describing mode shapes are known only for a restricted class of boundary conditions and for a limited set of models. For general boundary conditions and models, or systems involving coupling between a shell and an adjacent component (e.g., an acoustic field or a piezoceramic actuator), the modes must first be numerically approximated or experimentally determined before expansions can be formed.

Experimental determination of natural frequencies and modes is typically accomplished by exciting the structure using an acoustic source, shaker, magnetic driver, et cetera. At each natural frequency, the shape of the corresponding mode is determined and characterized for use in modal expansions. As detailed in [32,33], however, difficulties are encounted in this procedure in plate and shell-like structures due to the presence of multiple independent mode shapes which can occur at single experimental frequencies. In such cases, the experimentalist must excite the structure at various locations and complete an orthogonalization process to obtain a complete modal basis. The determination of which frequencies/modes to test for this behavior requires numerical analysis or extreme care when performing the experiments. Furthermore, as discussed in [32], internal or air damping will cause distortion of modes (e.g., modal lines that do not cross) which if not accounted for, will degrade modal expansions for approximating the structural dynamics.

In applications, modes determined for boundary conditions yielding analytic expressions (e.g., simply-supported end conditions) are occasionally used to approximate solutions to models with more general boundary conditions or models incorporating additional components. In some cases, the influence of various boundary conditions on modal characteristics has been investigated [17]; however, without any convergence analysis for such techniques, convergence 
of the numerical approximates to the true model solution cannot be guaranteed. Finally, it is difficult to apply modal techniques in applications modeled by nonlinear shell equations or nonlinear actuator contributions (e.g., electrostrictive actuators or piezoceramic actuators embedded in constrained damping layers).

Finite element approaches, on the other hand, are directly applicable for a variety of boundary and coupling conditions and in models including actuator contributions or nonlinear dynamics. Moreover, a large body of software exists for obtaining finite element solutions for various shell models (see [13, 20,21,22] for discussions of finite element methods and software for shells of revolution).

A difficulty when developing and applying finite element methods for shell applications, however, concerns the manifestation of various locking phenomena. Shear locking, which has also been studied extensively in the context of Reissner-Mindlin plate models, is due to element incompatibility when enforcing the Kirchhoff-Love constraint of vanishing transverse shear strains as the shell thickness $h$ tends to zero $[1,10]$. In shell applications, an even more serious problem leading to the failure of various finite element methods is the phenomenon of membrane or shear-membrane locking. This phenomenon occurs when the total deformation energy is bending dominated, and is due to smoothness and asymptotic constraints in the shell model which are not appropriately represented by the approximation method (see $[3,4,15,25$, 28]). If these constraints are not satisfied by approximating elements, the numerical solution is often overly stiff in the sense that the model exhibits bending dynamics which the approximate solution cannot match. As detailed in [25], mesh sizes must be chosen significantly smaller than the shell thickness to ensure accurate approximations with high-order finite elements in such bending dominated regimes. These examples also illustrate that even with such mesh size restrictions, low-order finite element methods often fail in such regimes. The use of finite elements which exhibit locking is detrimental in simulations and will seriously degrade the performance of any controllers designed around such methods.

A second issue which must be considered when developing finite element methods for shells concerns the piecewise constant material parameters and inexact boundary conditions which often arise in smart material applications. For example, the use of piezoceramic actuators leads to piecewise discontinuities in the density, stiffness, Poisson ratio and damping parameters. Consequently, finite element meshes must be aligned with the actuator boundaries to maintain accuracy (the reader is referred to [34] for a discussion of finite elements designed specifically for piezoelectric applications). In experiments involving shells, slight energy loss must often be modeled into the boundary conditions to account for inexact boundary clamps. Consequently, elements must be provided with extra degrees of freedom to accommodate these boundary conditions. Standard commercial codes not providing these capabilities will lead to potentially inaccurate results when applied to shells with smart material actuators and/or sensors.

The use of finite difference discretizations for approximating thin shell dynamics is less common due to inherent difficulties with the high-order equations and boundary conditions arising in the models. The reader is referred to [31] for further discussion of finite difference methods for shell applications.

In this paper, we consider a Galerkin method for discretizing thin shell models with linear or cubic splines chosen as basis elements for approximating the longitudinal and circumferential motion and cubic splines used to approximate the transverse component. These choices are motivated by smoothness and accuracy criteria, adaptability for a variety of boundary 
conditions, and suitability when including the contributions of actuators such as piezoceramic patches bonded to the shell [6]. For the discussion here, the shells are assumed to have lengths that are relatively short in relation to the radii, and the Donnell-Mushtari shell equations are employed. This facilitates the discussion while providing a framework which is easily extended to more accurate long shell models through the inclusion of Byrne-Flügge-Lur'ye components. In this study, the formulation of the method and numerical examples demonstrating the accuracy, efficiency and flexibility of the method are presented. Emphasis in these examples is placed on demonstrating that when $h_{x}$ denotes the axial mesh size, the expected $\mathcal{O}\left(h_{x}^{2}\right)$ and $\mathcal{O}\left(h_{x}^{4}\right)$ accuracy of the method is maintained when approximating with linear and cubic splines, respectively. Convergence analysis and further analysis of the method with regards to membrane and shear locking will appear in a future companion paper.

In Section 2, the strong and weak forms of the modeling equations for a thin shell with surface-mounted piezoceramic patches are summarized. Modal analysis for the special case of an undamped shell with constant coefficients and simply-supported boundary conditions is discussed in Section 3. This provides a framework for testing the convergence of the approximate mass and stiffness components in the system. The approximation method and resulting finite dimensional matrix system are detailed in Section 4. Finally, examples illustrating the method are presented in Section 5. These include modal approximations for various boundary conditions as well as results demonstrating convergence rates when approximating shell dynamics generated by various external inputs. To illustrate the shell dynamics generated by the piezoceramic patches, a modeled voltage spike to the patches is used as input in the final example. The resulting frequencies are then compared with those obtained in the eigenvalue (modal) analysis to demonstrate the consistency of the method.

\section{Donnell-Mushtari Equations}

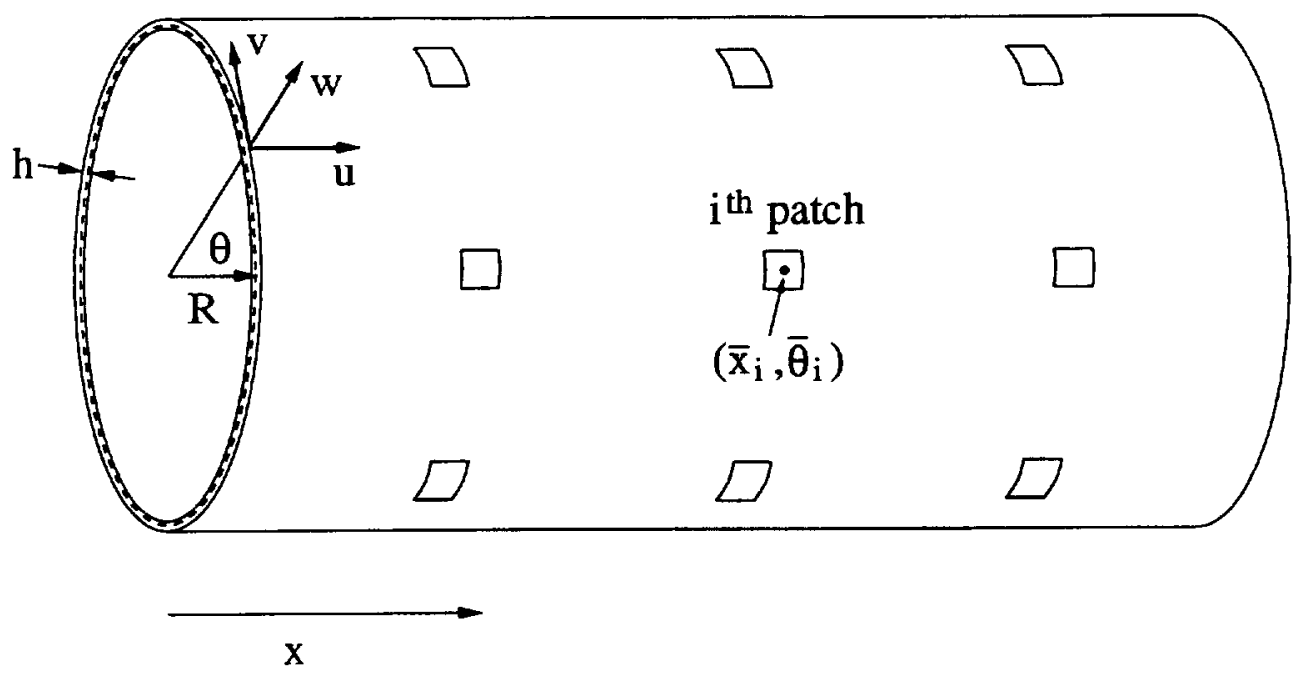

Figure 1. Thin cylindrical shell with surface mounted piezoceramic patches. 
We consider here a shell of length $\ell$, thickness $h$ and radius $R$ having mass density $\rho$, Young's modulus $E$, Poisson ratio $\nu$ and Kelvin-Voigt damping coefficient $c_{D}$. As depicted in Figure 1 , the axial direction is taken along the $x$-axis. The displacements of the middle surface in the longitudinal, circumferential and transverse directions are denoted by $u, v$ and $w$, respectively.

Bonded to the shell are $s$ pairs of piezoceramic patches which can be employed as sensors and/or actuators in structural and structural acoustic applications [5, 19]. To simplify the exposition, the patches are all assumed to have thickness $h_{p e}$, Young's modulus $E_{p e}$, Poisson ratio $\nu_{p e}$, and Kelvin-Voigt damping coefficient $c_{D_{p e}}$. Furthermore, it is assumed that the glue bonding layer provides negligible contribution to the structural dynamics. The reader is referred to to $[6,9]$ for details concerning the incorporation of differing patch characteristics and bonding layers in the ensuing models).

Throughout this discussion, it will be assumed that external inputs to the shell will be in the form of transverse, longitudinal and circumferential surface forces as well as line moments and forces generated by the patches. The surface forces can be used to model a variety of phenomena including coupling interactions with adjacent fields (e.g., acoustic fields - see [5]) and input from certain actuators. The patch moments and forces arise when the elements are used as actuators. While more general inputs can be considered, the above-mentioned cases demonstrate the flexibility of the numerical method for typical smart materials applications.

\subsection{Strong Form of Equations}

As detailed in $[6,26]$, moment and force balancing yields the Donnell-Mushtari equations

$$
\begin{gathered}
R \rho h \frac{\partial^{2} u}{\partial t^{2}}-R \frac{\partial N_{x}}{\partial x}-\frac{\partial N_{\theta x}}{\partial \theta}=R \hat{q}_{x}-R \sum_{i=1}^{s} \frac{\partial\left(N_{x}\right)_{p e_{i}}}{\partial x} \mathcal{S}_{p e_{i}}(x, \theta) \\
R \rho h \frac{\partial^{2} v}{\partial t^{2}}-\frac{\partial N_{\theta}}{\partial \theta}-R \frac{N_{x \theta}}{\partial x}=R \hat{q}_{\theta}-\sum_{i=1}^{s} \frac{\partial\left(N_{\theta}\right)_{p e_{i}}}{\partial \theta} \mathcal{S}_{p e_{i}}(x, \theta) \\
R \rho h \frac{\partial^{2} w}{\partial t^{2}}-R \frac{\partial^{2} M_{x}}{\partial x^{2}}-\frac{1}{R} \frac{\partial^{2} M_{\theta}}{\partial \theta^{2}}-2 \frac{\partial^{2} M_{x \theta}}{\partial x \partial \theta}+N_{\theta} \\
=R \hat{q}_{n}-\sum_{i=1}^{s}\left[R \frac{\partial^{2}\left(M_{x}\right)_{p e_{i}}}{\partial x^{2}}+\frac{1}{R} \frac{\partial^{2}\left(M_{\theta}\right)_{p e_{i}}}{\partial \theta^{2}}\right]
\end{gathered}
$$

as a model for the thin shell dynamics. Here $M_{x}, M_{\theta}, M_{x \theta}$, and $M_{\theta x}$ are internal moments, while $N_{x}, N_{\theta}, N_{x \theta}$ and $N_{\theta x}$ denote internal force resultants. External surface forces are denoted by $\hat{q}_{x}, \hat{q}_{\theta}, \hat{q}_{n}$ whereas the external resultants (line moments and forces) generated by the $i^{\text {th }}$ patch pair are designated by $\left(M_{x}\right)_{p e_{i}},\left(M_{\theta}\right)_{p e_{i}},\left(N_{x}\right)_{p e_{i}}$ and $\left(N_{\theta}\right)_{p e_{i}}$. The indicator function

$$
\mathcal{S}_{p e_{i}}(x, \theta) \equiv S_{1,2}(x) \hat{S}_{1,2}(\theta)
$$

where

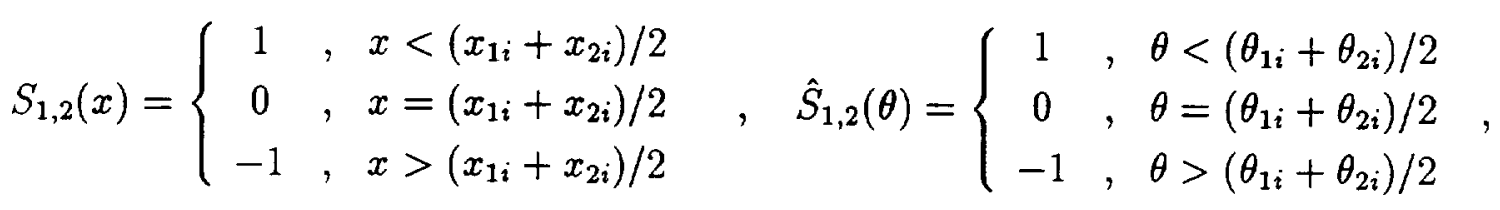


indicates the sense of the forces generated by the $i^{\text {th }}$ patch pair having edges at $x_{1 i}, x_{2 i}, \theta_{1 i}, \theta_{2 i}$. The symmetry of the function arises from the property that for homogeneous patches having uniform thickness, equal but opposite strains are generated about the point $\left(\bar{x}_{i}, \bar{\theta}_{i}\right)=$ $\left(\left(x_{1 i}+x_{2 i}\right) / 2,\left(\theta_{1 i}+\theta_{2 i}\right) / 2\right)$ in the two coordinate directions. Similarly, the characteristic function

$$
\chi_{p e_{i}}(x, \theta)= \begin{cases}1, & x_{1 i} \leq x \leq x_{2 i}, \theta_{1 i} \leq \theta \leq \theta_{2 i} \\ 0, & \text { otherwise }\end{cases}
$$

will be used in ensuing discussion to isolate internal and external contributions due to the $i^{\text {th }}$ patch pair.

Under the assumption that stress is proportional to a linear combination of strain and strain rate, the internal moments and force resultants in regions of the shell not covered by patches are given by

$$
\begin{aligned}
& N_{x}=\frac{E h}{\left(1-\nu^{2}\right)}\left[\frac{\partial u}{\partial x}+\frac{\nu}{R}\left(\frac{\partial v}{\partial \theta}+w\right)\right]+\frac{c_{D} h}{\left(1-\nu^{2}\right)} \frac{\partial}{\partial t}\left[\frac{\partial u}{\partial x}+\frac{\nu}{R}\left(\frac{\partial v}{\partial \theta}+w\right)\right] \\
& N_{\theta}=\frac{E h}{\left(1-\nu^{2}\right)}\left[\frac{1}{R} \frac{\partial v}{\partial \theta}+\frac{w}{R}+\nu \frac{\partial u}{\partial x}\right]+\frac{c_{D} h}{\left(1-\nu^{2}\right)} \frac{\partial}{\partial t}\left[\frac{1}{R} \frac{\partial v}{\partial \theta}+\frac{w}{R}+\nu \frac{\partial u}{\partial x}\right] \\
& N_{x \theta}=N_{\theta x}=\frac{E h}{2(1+\nu)}\left[\frac{\partial v}{\partial x}+\frac{1}{R} \frac{\partial u}{\partial \theta}\right]+\frac{c_{D} h}{2(1+\nu)} \frac{\partial}{\partial t}\left[\frac{\partial v}{\partial x}+\frac{1}{R} \frac{\partial u}{\partial \theta}\right] \\
& M_{x}=\frac{-E h^{3}}{12\left(1-\nu^{2}\right)}\left[\frac{\partial^{2} w}{\partial x^{2}}+\frac{\nu}{R^{2}} \frac{\partial^{2} w}{\partial \theta^{2}}\right]-\frac{c_{D} h^{3}}{12\left(1-\nu^{2}\right)} \frac{\partial}{\partial t}\left[\frac{\partial^{2} w}{\partial x^{2}}+\frac{\nu}{R^{2}} \frac{\partial^{2} w}{\partial \theta^{2}}\right] \\
& M_{\theta}=\frac{-E h^{3}}{12\left(1-\nu^{2}\right)}\left[\frac{1}{R^{2}} \frac{\partial^{2} w}{\partial \theta^{2}}+\nu \frac{\partial^{2} w}{\partial x^{2}}\right]-\frac{c_{D} h^{3}}{12\left(1-\nu^{2}\right)} \frac{\partial}{\partial t}\left[\frac{1}{R^{2}} \frac{\partial^{2} w}{\partial \theta^{2}}+\nu \frac{\partial^{2} w}{\partial x^{2}}\right] \\
& M_{x \theta}=M_{\theta x}=\frac{-E h^{3}}{12 R(1+\nu)} \frac{\partial^{2} w}{\partial x \partial \theta}-\frac{c_{D} h^{3}}{12 R(1+\nu)} \frac{\partial}{\partial t}\left[\frac{\partial^{2} w}{\partial x \partial \theta}\right]
\end{aligned}
$$

(see [6] or [26] for the undamped case). The substitution of these equations in (2.1) yields the damped form of the Donnell-Mushtari equations for a uniform, homogeneous shell that is devoid of patches.

The bonding of piezoceramic patches to the shell produces contributions due to both internal (material) and external moments and forces. The internal contributions are due to the geometrical and material changes afforded by the patches. The external contributions result from the piezoelectric effect in the patches which is manifested as generated strains in response to applied voltages.

As detailed in $[6,9]$, the internal force resultant $N_{x}$ is given by

$$
\begin{aligned}
& N_{x}=\frac{E h}{1-\nu^{2}}\left[\frac{\partial u}{\partial x}+\frac{\nu}{R}\left(\frac{\partial v}{\partial \theta}+w\right)\right]+\sum_{i=1}^{s} \frac{2 E_{p e} h_{p e}}{1-\nu_{p e}^{2}}\left[\frac{\partial u}{\partial x}+\frac{\nu_{p e}}{R}\left(\frac{\partial v}{\partial \theta}+w\right)\right] \chi_{p e_{i}}(x, \theta) \\
& +\frac{c_{D} h}{1-\nu^{2}} \frac{\partial}{\partial t}\left[\frac{\partial u}{\partial x}+\frac{\nu}{R}\left(\frac{\partial v}{\partial \theta}+w\right)\right]+\sum_{i=1}^{s} \frac{2 c_{D p e} h_{p e}}{1-\nu_{p e}^{2}} \frac{\partial}{\partial t}\left[\frac{\partial u}{\partial x}+\frac{\nu_{p e}}{R}\left(\frac{\partial v}{\partial \theta}+w\right)\right] \chi_{p e_{i}}(x, \theta)
\end{aligned}
$$


for systems in which the patches have identical properties (compare with (2.2)). Analogous expressions are given in $[6,9]$ for the remaining resultants and the resultants when the patches have differing material characteristics.

To characterize the external contributions, it is typical to start with the assumption that the strains generated by a patch are proportional to the applied voltage [6]. Since differing voltages can be applied to the outer and inner patches in the pair, we will differentiate between the two with $V_{i 1}(t)$ and $V_{i 2}(t)$ used to denote the voltages to the outer and inner patches in the $i^{\text {th }}$ pair, respectively. The proportionality constant relating generated strain to the input voltage is designated by $d_{31}$. As detailed in [6], the total external moments and forces generated by the patches are

$$
\begin{aligned}
& \left(M_{x}\right)_{p e_{i}}=\left[\left(M_{x}\right)_{p e_{i 1}}+\left(M_{x}\right)_{p e_{i 2}}\right] \chi_{p e_{i}}(x, \theta) \\
& \left(M_{\theta}\right)_{p e_{i}}=\left[\left(M_{\theta}\right)_{p e_{i 1}}+\left(M_{\theta}\right)_{p e_{i 2}}\right] \chi_{p e_{i}}(x, \theta) \\
& \left(N_{x}\right)_{p e_{i}}=\left[\left(N_{x}\right)_{p e_{i 1}}+\left(N_{x}\right)_{p e_{i 2}}\right] \chi_{p e_{i}}(x, \theta) \mathcal{S}_{p e_{i}}(x, \theta) \\
& \left(N_{\theta}\right)_{p e_{i}}=\left[\left(N_{\theta}\right)_{p e_{i 1}}+\left(N_{\theta}\right)_{p e_{i 2}}\right] \chi_{p e_{i}}(x, \theta) \mathcal{S}_{p e_{i}}(x, \theta)
\end{aligned}
$$

where

$$
\begin{aligned}
& \left(M_{x}\right)_{p e_{i 1}}=\frac{-E_{p e}}{1-\nu_{p e}}\left[\frac{1}{8}\left(4\left(\frac{h}{2}+h_{p e}\right)^{2}-h^{2}\right)+\frac{1}{R} \frac{1}{24}\left(8\left(\frac{h}{2}+h_{p e}\right)^{3}-h^{3}\right)\right] \frac{d_{31}}{h_{p e}} V_{i 1} \\
& \left(M_{x}\right)_{p e_{i 2}}=\frac{E_{p e}}{1-\nu_{p e}}\left[\frac{1}{8}\left(4\left(\frac{h}{2}+h_{p e}\right)^{2}-h^{2}\right)-\frac{1}{R} \frac{1}{24}\left(8\left(\frac{h}{2}+h_{p e}\right)^{3}-h^{3}\right)\right] \frac{d_{31}}{h_{p e}} V_{i 2} \\
& \left(M_{\theta}\right)_{p e_{11}}=\frac{-E_{p e}}{1-\nu_{p e}}\left[\frac{1}{8}\left(4\left(\frac{h}{2}+h_{p e}\right)^{2}-h^{2}\right)\right] \frac{d_{31}}{h_{p e}} V_{i 1} \\
& \left(M_{\theta}\right)_{p e_{i 2}}=\frac{E_{p e}}{1-\nu_{p e}}\left[\frac{1}{8}\left(4\left(\frac{h}{2}+h_{p e}\right)^{2}-h^{2}\right)\right] \frac{d_{31}}{h_{p e}} V_{i 2} \\
& \left(N_{x}\right)_{p e_{i 1}}=\frac{-E_{p e}}{1-\nu_{p e}}\left[h_{p e}+\frac{1}{R} \frac{1}{8}\left(4\left(\frac{h}{2}+h_{p e}\right)^{2}-h^{2}\right)\right] \frac{d_{31}}{h_{p e}} V_{i 1} \\
& \left(N_{x}\right)_{p e_{i 2}}=\frac{-E_{p e}}{1-\nu_{p e}}\left[h_{p e}-\frac{1}{R} \frac{1}{8}\left(4\left(\frac{h}{2}+h_{p e}\right)^{2}-h^{2}\right)\right] \frac{d_{31}}{h_{p e}} V_{i 2} \\
& \left(N_{\theta}\right)_{p e_{i 1}}=\frac{-E_{p e}}{1-\nu_{p e}} d_{31} V_{i 1} \\
& \left(N_{\theta}\right)_{p e_{i 2}}=\frac{-E_{p e}}{1-\nu_{p e}} d_{31} V_{i 2} .
\end{aligned}
$$

When substituted into (2.1), the expressions (2.4) provide the input from the patches when voltages are applied. 


\subsection{Boundary Conditions}

Appropriate boundary conditions are dictated by the experimental setup or application under consideration. In many setups, such as the experimental shell apparatus at NASA Langley Research Center, the shell is supported by heavy endcaps. In such cases, the fixed-edge conditions

$$
u=v=w=\frac{\partial w}{\partial x}=0 \quad, \quad x=0, \ell
$$

may adequately model the end behavior of the shell.

If slight boundary movement or rotations are suspected, "almost fixed" boundary conditions of the type discussed in $[7,8]$ can be employed. Such boundary conditions can be used to model the slight energy which result due to imperfect clamping of the structure.

A third type of commonly considered boundary conditions are the simply-supported or shear diaphragm edge conditions

$$
v=w=M_{x}=N_{x}=0 \quad, \quad x=0, \ell .
$$

These boundary conditions are theoretically attractive since they provide one of the few situations in which explicit modal expansions can be calculated for the Donnell-Mushtari shell equations. They are of limited use when modeling experimental shells, however, since they are appropriate only for endcaps which prevent movement in the $v$ and $w$ directions but are sufficiently flexible in the $x$-direction so that negligible moments $M_{x}$ and forces $N_{x}$ are generated.

The Galerkin method of this work is equally efficient to implement for models incorporating the fixed-edge boundary conditions (2.6), "almost fixed" boundary conditions, or the simplesupported edge conditions (2.7). The method is demonstrated for the fixed-edge and simplysupported conditions while modifications to adapt the method to "almost fixed" can be found in $[7,8]$. Later discussion will also illustrate the manner through which the method can be adapted to alternative boundary conditions which many arise in physical applications.

\subsection{Weak Form of Equations}

As noted in (2.1), the strong form of the modeling equations involves first and second derivatives of both the moment and force resultants. For structures with surface-mounted or embedded actuators or sensors (e.g., piezoceramic patches), this differentiation leads to difficulties due to discontinuities in the resultants. The internal resultants contain piecewise discontinuities due to material changes introduced by the patches (see for example, (2.3)). The external patch contributions are also discontinuous since they are defined only over regions covered by patches (see (2.4)). As a result, formal analysis and approximation using the strong form of the modeling equations leads to derivatives of the Dirac delta "function."

To alleviate these difficulties, we consider a weak form of the modeling equations. As detailed in [6], such equations can be derived from energy considerations and are equivalent to the strong form of the modeling equations under suitable smoothness conditions. This yields a form of the model which facilitates well-posedness analysis [5] and eliminates the difficulties associated with the discontinuous resultants. Moreover, smoothness requirements on basis functions are reduced which proves advantageous when constructing system matrices. 
The weak form of (2.1), as derived from energy considerations in [6], is given by

$$
\begin{gathered}
\int_{0}^{2 \pi} \int_{0}^{\ell}\left\{R \rho h \frac{\partial^{2} u}{\partial t^{2}} \eta_{1}+R N_{x} \frac{\partial \eta_{1}}{\partial x}+N_{\theta x} \frac{\partial \eta_{1}}{\partial \theta}-R \hat{q}_{x} \eta_{1}-R \sum_{i=1}^{s}\left(N_{x}\right)_{p e_{i}} \frac{\partial \eta_{1}}{\partial x}\right\} d x d \theta=0 \\
\int_{0}^{2 \pi} \int_{0}^{\ell}\left\{R \rho h \frac{\partial^{2} v}{\partial t^{2}} \eta_{2}+N_{\theta} \frac{\partial \eta_{2}}{\partial \theta}+R N_{x \theta} \frac{\partial \eta_{2}}{\partial x}-R \hat{q}_{\theta} \eta_{2}-\sum_{i=1}^{s}\left(N_{\theta}\right)_{p e_{i}} \frac{\partial \eta_{2}}{\partial \theta}\right\} d x d \theta=0 \\
\int_{0}^{2 \pi} \int_{0}^{\ell}\left\{R \rho h \frac{\partial^{2} w}{\partial t^{2}} \eta_{3}+N_{\theta} \eta_{3}-R M_{x} \frac{\partial^{2} \eta_{3}}{\partial x^{2}}-\frac{1}{R} M_{\theta} \frac{\partial^{2} \eta_{3}}{\partial \theta^{2}}-2 M_{x \theta} \frac{\partial^{2} \eta_{3}}{\partial x \partial \theta}\right. \\
\left.-R \hat{q}_{n} \eta_{3}+\sum_{i=1}^{s}\left[R\left(M_{x}\right)_{p e_{i}} \frac{\partial^{2} \eta_{3}}{\partial x^{2}}+\frac{1}{R}\left(M_{\theta}\right)_{p e_{i}} \frac{\partial^{2} \eta_{3}}{\partial \theta^{2}}\right]\right\} d x d \theta=0
\end{gathered}
$$

for all $\vec{\eta}=\left[\eta_{1}, \eta_{2}, \eta_{3}\right] \in V$ where $V$ denotes the space of test functions (as detailed in [6], the indicator functions appear only in the definition of the external force resultants in the weak form of the equations). Specific choices for $V$ depend upon the boundary conditions. For the fixed-edge boundary conditions (2.6), the space of test functions is taken to be

$$
V=H_{0}^{1}(\Omega) \times H_{0}^{1}(\Omega) \times H_{0}^{2}(\Omega)
$$

where

$$
\begin{aligned}
& H_{0}^{1}(\Omega)=\left\{\eta \in H^{1}(\Omega): \eta(0)=\eta(\ell)=0\right\} \\
& H_{0}^{2}(\Omega)=\left\{\eta \in H^{2}(\Omega): \eta(0)=\eta_{x}(0)=\eta(\ell)=\eta_{x}(\ell)=0\right\} .
\end{aligned}
$$

In the case of the simply-supported edge conditions (2.7), essential boundary conditions are imposed only on the circumferential and transverse functions and $V$ is taken to be

$$
V=H^{1}(\Omega) \times H_{0}^{1}(\Omega) \times H_{L}^{2}(\Omega)
$$

with

$$
H_{L}^{2}(\Omega)=\left\{\eta \in H^{2}(\Omega): \eta(0)=\eta(\ell)=0\right\} .
$$

In general, $V$ is simply taken as the subset of the traditional Sobolev spaces satisfying essential boundary conditions (the reader is referred to [7] for modifications to account for "almost fixed" boundary conditions).

A comparison between (2.8) and (2.1) illustrates that in the weak form, derivatives are transferred from the discontinuous resultants onto suitably smooth test functions. This alleviates the difficulties associated with the discontinuities and reduces smoothness requirements on approximate solutions. 


\section{Modal Solution - Simply-Supported Boundary Con- ditions, Constant Coefficients, No Damping}

As noted in the introduction, closed form modal expressions for shell models can be obtained only in a limited number of cases. One case in which analytic frequencies and modes can be calculated is the undamped $\left(c_{D}=0\right)$ shell model with simply-supported edge conditions (2.7) and constant parameters $\rho, \nu$ and $E$. Separation of variables in this setting is classical and can be found in numerous texts (e.g., [27]); we summarize the arguments here to facilitate numerical examples in later sections. While such conditions are not attained in typical shell applications, the consideration of the undamped, constant coefficient shell model with simply-supported edge conditions provides an excellent means of testing discretization techniques since approximate solutions can be compared with analytic values. The discretization techniques can then be used to approximate natural frequencies, modes and shell dynamics in models which incorporate piecewise constant parameters (including damping) and physically realistic boundary conditions.

Throughout this section, it is assumed that $c_{D}=0$ and $\rho, \nu$ and $E$ are constant. Since our interest here is restricted to the calculation of natural frequencies and modes, we will also consider the shell model to be unforced (no external patch contributions and $\hat{q}_{x}=\hat{q}_{\theta}=\hat{q}_{n}=0$ ). In this case, the strong form of the Donnell-Mushtari thin shell equations (2.1) can be written in the operator format

$$
\rho h \frac{\partial^{2} \vec{u}}{\partial t^{2}}=L \vec{u}
$$

where $\vec{u}=[u, v, w]^{T}$. The operator $L$ here is given by

$$
L=\frac{E h}{1-\nu^{2}}\left[\begin{array}{ccc}
\frac{\partial^{2}}{\partial x^{2}}+\frac{(1-\nu)}{2 R^{2}} \frac{\partial^{2}}{\partial \theta^{2}} & \frac{(1+\nu)}{2 R} \frac{\partial^{2}}{\partial x \partial \theta} & \frac{\nu}{R} \frac{\partial}{\partial x} \\
\frac{(1+\nu)}{2 R} \frac{\partial^{2}}{\partial x \partial \theta} & \frac{(1-\nu)}{2} \frac{\partial^{2}}{\partial x^{2}}+\frac{1}{R^{2}} \frac{\partial^{2}}{\partial \theta^{2}} & \frac{1}{R^{2}} \frac{\partial}{\partial \theta} \\
-\frac{\nu}{R} \frac{\partial}{\partial x} & -\frac{1}{R^{2}} \frac{\partial}{\partial \theta} & -\frac{1}{R^{2}}-k\left[\frac{\partial^{4}}{\partial x^{4}}+\frac{2}{R^{2}} \frac{\partial^{4}}{\partial x^{2} \partial \theta^{2}}+\frac{1}{R^{4}} \frac{\partial^{4}}{\partial \theta^{4}}\right]
\end{array}\right]
$$

where $k=h^{2} / 12$. Boundary conditions for the shell are denoted by

$$
B \vec{u}=\overrightarrow{0} .
$$

Due to linearity, the displacements are expressed as

$$
\begin{aligned}
& u(t, x, \theta)=T(t) U(x, \theta) \\
& v(t, x, \theta)=T(t) V(x, \theta) \\
& w(t, x, \theta)=T(t) W(x, \theta),
\end{aligned}
$$

and spatial and temporal components are separated to yield the eigenvalue problem

$$
\begin{aligned}
& L \vec{U}+\rho h \omega^{2} \vec{U}=\overrightarrow{0} \\
& B \vec{U}=\overrightarrow{0}
\end{aligned}
$$


along with the temporal equation $T^{\prime \prime}+\omega^{2} T=0$. Here $\omega$ is the circular frequency of vibration and $\vec{U}=[U, V, W]^{T}$ contains the vibration modes in the axial, circumferential and transverse directions. We point out that separating variables to arrive at the eigenvalue problem (3.2) is equivalent to the assumption of a harmonic response in all components and one obtains the same eigenvalue problem in both cases.

The structure of the eigenfunctions $U, V$ and $W$ is dependent upon the boundary conditions with closed form expressions attainable in only a few cases. To illustrate, we consider the eigenvalue problem (3.2) with the simply-supported boundary conditions (2.7). One form of the natural vibration modes for this case is

$$
\begin{aligned}
& U(x, \theta)=A_{1} \cos \left(\frac{n \pi x}{\ell}\right) \cos (m \theta) \\
& V(x, \theta)=B_{1} \sin \left(\frac{n \pi x}{\ell}\right) \sin (m \theta) \\
& W(x, \theta)=C_{1} \sin \left(\frac{n \pi x}{\ell}\right) \cos (m \theta) .
\end{aligned}
$$

The choice of the cosine representation for the $x$ component of the axial vibration and sines for the circumferential and radial vibrations guarantees that the modes satisfy the boundary conditions. The relationship between the circumferential and axial/radial vibrations dictates that the former must be $90^{\circ}$ out-of-phase from the latter two in $\theta$ (see the moment and force expressions (2.2) or operator definition (3.1)).

To determine the relationship between the frequencies $\omega$ and the wave numbers $m$ and $n$, the expressions (3.3) are substituted into (3.2) to yield the system

$$
\left[\begin{array}{ccc}
\Omega^{2}-H_{1} & \frac{\lambda m}{2}(1+\nu) & \nu \lambda \\
\frac{\lambda m}{2}(1+\nu) & \Omega^{2}-H_{2} & -m \\
\nu \lambda & -m & \Omega^{2}-H_{3}
\end{array}\right]\left[\begin{array}{c}
A_{1} \\
B_{1} \\
C_{1}
\end{array}\right]=\left[\begin{array}{l}
0 \\
0 \\
0
\end{array}\right]
$$

where

$$
\begin{aligned}
& H_{1}=\lambda^{2}+\frac{m^{2}}{2}(1-\nu) \\
& H_{2}=\frac{\lambda^{2}}{2}(1-\nu)+m^{2} \\
& H_{3}=1+\tilde{k}\left(\lambda^{2}+m^{2}\right)^{2}
\end{aligned}
$$

and

$$
\begin{aligned}
& \Omega^{2}=\frac{\rho}{E}\left(1-\nu^{2}\right) \omega^{2} R^{2} \\
& \lambda=\frac{n \pi R}{L} \\
& \tilde{k}=\frac{k}{R^{2}}=\frac{h^{2}}{12 R^{2}} .
\end{aligned}
$$

A nontrivial solution is determined by setting the determinant to zero. This yields the cubic equation

$$
\Omega^{6}-K_{2} \Omega^{4}+K_{1} \Omega^{2}-K_{0}=0
$$


in $\Omega^{2}$. The coefficients here are given by

$$
\begin{aligned}
& K_{2}=1+\frac{1}{2}(3-\nu)\left(m^{2}+\lambda^{2}\right)+\tilde{k}\left(m^{2}+\lambda^{2}\right)^{2} \\
& K_{1}=\frac{1}{2}(1-\nu)\left[(3+2 \nu) \lambda^{2}+m^{2}+\left(m^{2}+\lambda^{2}\right)^{2}+\frac{3-\nu}{1-\nu} \tilde{k}\left(m^{2}+\lambda^{2}\right)^{3}\right] \\
& K_{0}=\frac{1}{2}(1-\nu)\left[\left(1-\nu^{2}\right) \lambda^{4}+\tilde{k}\left(m^{2}+\lambda^{2}\right)^{4}\right] .
\end{aligned}
$$

The solutions to this cubic equation are then given by

$$
\Omega_{m n j}^{2}=-\frac{2}{3} \sqrt{K_{2}^{2}-3 K_{1}} \cos \left(\frac{\alpha+2(j-1) \pi}{3}\right)+\frac{K_{2}}{3} \quad, \quad j=1,2,3
$$

where

$$
\alpha=\cos ^{-1}\left(\frac{-27 K_{0}-2 K_{2}^{3}+9 K_{1} K_{2}}{2 \sqrt{\left(K_{2}^{2}-3 K_{1}\right)^{3}}}\right) .
$$

Finally, the natural frequencies for the shell, with units of hertz, are given by

$$
f_{m n j}=\frac{\Omega_{m n j}}{2 \pi R} \sqrt{\frac{E}{\rho\left(1-\nu^{2}\right)}} \quad, \quad j=1,2,3 .
$$

We point out that due to the cubic nature of the characteristic equation, three natural frequencies and mode shapes are obtained for each set of wave numbers $m$ and $n$. This leads to a significant interlacing of frequencies.

A second set of modes which satisfy the boundary conditions and $\theta$ compatibility criteria is

$$
\begin{aligned}
& U(x, \theta)=A_{2} \cos \left(\frac{n \pi x}{\ell}\right) \sin (m \theta) \\
& V(x, \theta)=B_{2} \sin \left(\frac{n \pi x}{\ell}\right) \cos (m \theta) \\
& W(x, \theta)=C_{2} \sin \left(\frac{n \pi x}{\ell}\right) \sin (m \theta) .
\end{aligned}
$$

The system which arises when using this form of the modes is

$$
\left[\begin{array}{ccc}
\Omega^{2}-H_{1} & -\frac{\lambda m}{2}(1+\nu) & \nu \lambda \\
-\frac{\lambda m}{2}(1+\nu) & \Omega^{2}-H_{2} & m \\
\nu \lambda & m & \Omega^{2}-H_{3}
\end{array}\right]\left[\begin{array}{c}
A_{2} \\
B_{2} \\
C_{2}
\end{array}\right]=\left[\begin{array}{l}
0 \\
0 \\
0
\end{array}\right]
$$

which differs in sign in the $(1,2),(2,1),(2,3)$ and $(3,2)$ elements from $(3.4)$ which was obtained using the first set of modal expansions. The characteristic equations, however, are the same in both cases and are given by (3.5). Hence both sets of expressions yield the same natural frequencies for the shell. This should be expected since the second set simply represents a phase shift in $\theta$ of the first. For this reason, most authors consider only the first set of vibration modes.

In some instances, however, the second set (3.7) contributes linearly independent modes and hence must be retained in order to obtain a complete basis for approximation. This is illustrated in the examples of Section 5.1. Further details regarding the modal analysis can be found in [16]. 


\section{Approximation Method}

To approximate the solutions $u, v, w$ to the system (2.8) or eigenvalue problem (3.2), basis functions $\mathcal{B}_{u_{k}}(\theta, x), \mathcal{B}_{v_{k}}(\theta, x)$ and $\mathcal{B}_{w_{k}}(\theta, x)$ in $V$ are chosen and used to form expansions

$$
\begin{aligned}
& u^{N}(t, \theta, x)=\sum_{k=1}^{\mathcal{N}_{u}} u_{k}(t) \mathcal{B}_{u_{k}}(\theta, x) \\
& v^{N}(t, \theta, x)=\sum_{k=1}^{\mathcal{N}_{v}} v_{k}(t) \mathcal{B}_{v_{k}}(\theta, x) \\
& w^{N}(t, \theta, x)=\sum_{k=1}^{\mathcal{N}_{w}} w_{k}(t) \mathcal{B}_{w_{k}}(\theta, x) .
\end{aligned}
$$

The time-dependent generalized Fourier coefficients $u_{k}(t), v_{k}(t), w_{k}(t)$ are then determined by orthogonalizing the residual with respect to linearly independent test functions from the span of the bases.

To exploit the tensor nature of the cylindrical shell domain $\Omega$ and periodicity in $\theta$, the bases for the three displacements are constructed with Fourier components in $\theta$ and spline components in $x$. When expressed with real components, this yields

$$
\begin{aligned}
u^{N}(t, \theta, x) & =\sum_{n=1}^{\hat{N}_{u}} u_{0 n}(t) B_{u_{n}}(x) \\
& +\sum_{m=1}^{M_{u}} \sum_{n=1}^{\hat{N}_{u}} u_{m n}(t) \cos (m \theta) B_{u_{n}}(x)+\sum_{m=1}^{M_{u}} \sum_{n=1}^{\hat{N}_{u}} \tilde{u}_{m n}(t) \sin (m \theta) B_{u_{n}}(x)
\end{aligned}
$$

with similar expressions for $v^{N}(t, \theta, x)$ and $w^{N}(t, \theta, x)$. In the expansions, $M_{u}, M_{v}, M_{w}$ are the Fourier limits and $\hat{N}_{u}, \hat{N}_{v}, \hat{N}_{w}$ denote the number of splines $B_{u_{n}}(x), B_{v_{n}}(x), B_{w_{n}}(x)$ used when approximating the longitudinal, circumferential and transverse displacements, respectively. The total number of basis functions for each component is $\mathcal{N}_{u}=\hat{N}_{u} \cdot\left(2 M_{u}+1\right), \mathcal{N}_{v}=\hat{N}_{v}$. $\left(2 M_{v}+1\right)$ and $\mathcal{N}_{w}=\hat{N}_{w} \cdot\left(2 M_{w}+1\right)$.

\subsection{Axial Basis Functions}

The choice of splines in the axial variable is motivated by the following criteria:

(i) efficiency;

(ii) flexibility with regard to internal and external patch contributions;

(iii) adaptability with respect to various boundary conditions;

(iv) accuracy.

For the longitudinal and circumferential displacements, both linear and cubic spline bases are considered. Due to differentiability requirements, only cubic splines are used when approximating the transverse displacement.

In all cases, a uniform partition along the $x$-axis is considered with gridpoints $x_{n}=$ $n h_{x}, h_{x}=\ell / N, n=1, \cdots, N$. For $n=-1,0,1, \cdots, N+1$, standard cubic splines are defined by 


$$
b_{n}(x)=\frac{1}{h_{x}^{3}} \begin{cases}\left(x-x_{n-2}\right)^{3} & , x \in\left[x_{n-2}, x_{n-1}\right] \\ h_{x}^{3}+3 h_{x}^{2}\left(x-x_{n-1}\right)+3 h_{x}\left(x-x_{n-1}\right)^{2}-3\left(x-x_{n-2}\right)^{3} & , x \in\left[x_{n-1}, x_{n}\right] \\ h_{x}^{3}+3 h_{x}^{2}\left(x_{n+1}-x\right)+3 h_{x}\left(x_{n+1}-x\right)^{2}-3\left(x_{n+1}-x\right)^{3} & , x \in\left[x_{n}, x_{n+1}\right] \\ \left(x_{n+2}-x\right)^{3} & , x \in\left[x_{n+1}, x_{n+2}\right] \\ 0 & , \text { otherwise }\end{cases}
$$

(see [29]). The standard linear splines are defined by

$$
c_{n}(x)=\frac{1}{h_{x}}\left\{\begin{array}{ll}
\left(x-x_{n-1}\right) & , x \in\left[x_{n-1}, x_{n}\right] \\
\left(x_{n+1}-x\right) & , x \in\left[x_{n}, x_{n+1}\right] \\
0 & , \text { otherwise }
\end{array},\right.
$$

for $n=0, \cdots, N$.

In both cases, the standard splines must be modified to satisfy the essential boundary conditions to ensure approximate solutions in $V$. These modifications are summarized as follows.

(i) Fixed displacements at $x=0, \ell$ :

To construct basis functions satisfying fixed displacements (but unspecified slopes) at $x=0, \ell$, the modified cubic splines are taken to be

$$
\hat{b}_{n}(x)=\left\{\begin{array}{ll}
b_{0}(x)-4 b_{-1}(x) & , n=0 \\
b_{1}(x)-b_{-1}(x) & , n=1 \\
b_{n}(x) & , n=2, \cdots, N-2, \\
b_{N-1}(x)-b_{N+1} & , n=N-1 \\
b_{N}(x)-4 b_{N+1}(x) & , n=N
\end{array},\right.
$$

for a total of $N+1$ functions. To satisfy the same condition, the first and last linear splines are omitted from the set to yield

$$
\hat{c}_{n}(x)=c_{n}(x), n=1, \cdots, N-1 .
$$

It should be noted that with these definitions, $\hat{b}_{n}(0)=\hat{b}_{n}(\ell)=0$ and $\hat{c}_{n}(0)=\hat{c}_{n}(\ell)=0$.

(ii) Fixed displacements and slope at $x=0, \ell$ :

Only the cubic splines are required to satisfy a condition of fixed displacement and slope at the endpoints since this is a condition imposed only on the transverse displacements. In this case, the modified splines are taken to be

$$
\tilde{b}_{n}(x)= \begin{cases}b_{0}(x)-2 b_{-1}(x)-2 b_{1}(x) & , n=1 \\ b_{n}(x) & , n=2, \cdots, N-2, \\ b_{N}(x)-2 b_{N-1}(x)-2 b_{N+1}(x) & , n=N-1\end{cases}
$$

for a total of $N-1$ basis functions. Note that these functions satisfy

$$
\tilde{b}_{n}(0)=\tilde{b}_{n}^{\prime}(0)=\tilde{b}_{n}(\ell)=\tilde{b}_{n}^{\prime}(\ell)=0 \text {. }
$$




\subsection{Bases for Specific Boundary Conditions}

Appropriate bases for various boundary conditions are then constructed by considering modified splines satisfying any essential boundary conditions. For example, the bases used for simply-supported shells (2.7) must satisfy the conditions $B_{v_{n}}(0)=B_{v_{n}}(\ell)=0, B_{w_{n}}(0)=$ $B_{w_{n}}(\ell)=0$ (the moment and shear conditions are natural and hence do not need to be explicitly enforced). Choices for shell models with fixed-edge conditions are summarized in Table 1 while corresponding choices for simply-supported edge conditions are given in Table 2. The last column in each table summarizes the total number of axial functions in each expansion.

Once the axial bases are chosen, they are combined with the Fourier components in $\theta$ to form the total bases. We reiterate that the basis limits in each case are given by $\mathcal{N}_{u}=$ $\hat{N}_{u} \cdot\left(2 M_{u}+1\right), \mathcal{N}_{v}=\hat{N}_{v} \cdot\left(2 M_{v}+1\right)$ and $\mathcal{N}_{w}=\hat{N}_{w} \cdot\left(2 M_{w}+1\right)$.

We point out that the linear and cubic spline bases described here are but two choices from among many that can be made for the axial components. For the applications considered here, cubic splines provided a good balance between accuracy, efficiency and adaptability with regards to patches and boundary conditions. If higher accuracy is desired, however (with slightly more expense when constructing system matrices), quintic splines can be employed. Similarly, spectral expansions employing Legendre, Chebyshev or sinc functions can be employed once modifications have been made for boundary conditions.

\begin{tabular}{r|c|l|l}
\hline Shell Component & Axial Basis Functions & Component Definition & Axial Limit \\
\hline longitudinal - linear & $B_{u_{n}}(x)=\hat{c}_{n}(x)$ & $\hat{c}_{n}(x)$ defined in $(4.6)$ & $\hat{N}_{u}=N_{u}-1$ \\
cubic & $B_{u_{n}}(x)=\hat{b}_{n}(x)$ & $\hat{b}_{n}(x)$ defined in $(4.5)$ & $\hat{N}_{u}=N_{u}+1$ \\
\hline circumferential - linear & $B_{v_{n}}(x)=\hat{c}_{n}(x)$ & $\hat{c}_{n}(x)$ defined in (4.6) & $\hat{N}_{v}=N_{v}-1$ \\
cubic & $B_{v_{n}}(x)=\hat{b}_{n}(x)$ & $\hat{b}_{n}(x)$ defined in $(4.5)$ & $\hat{N}_{v}=N_{v}+1$ \\
\hline transverse - cubic & $B_{w_{n}}(x)=\tilde{b}_{n}(x)$ & $\tilde{b}_{n}(x)$ defined in $(4.7)$ & $\hat{N}_{w}=N_{w}-1$ \\
\hline
\end{tabular}

Table 1. Axial basis definitions for models with fixed-edge boundary conditions (2.6).

\begin{tabular}{r|c|l|l}
\hline Shell Component & Axial Basis Functions & Component Definition & Axial Limit \\
\hline longitudinal - linear & $B_{u_{n}}(x)=c_{n}(x)$ & $c_{n}(x)$ defined in $(4.4)$ & $\hat{N}_{u}=N_{u}+1$ \\
cubic & $B_{u_{n}}(x)=b_{n}(x)$ & $b_{n}(x)$ defined in $(4.3)$ & $\hat{N}_{u}=N_{u}+3$ \\
\hline circumferential - linear & $B_{v_{n}}(x)=\hat{c}_{n}(x)$ & $\hat{c}_{n}(x)$ defined in (4.6) & $\hat{N}_{v}=N_{v}-1$ \\
cubic & $B_{v_{n}}(x)=\hat{b}_{n}(x)$ & $\hat{b}_{n}(x)$ defined in (4.5) & $\hat{N}_{v}=N_{v}+1$ \\
\hline transverse - cubic & $B_{w_{n}}(x)=\hat{b}_{n}(x)$ & $\hat{b}_{n}(x)$ defined in (4.5) & $\hat{N}_{w}=N_{w}+1$ \\
\hline
\end{tabular}

Table 2. Axial basis definitions for models with simply-supported boundary conditions (2.7). 


\subsection{Components in the Finite Dimensional System}

With bases thus defined, approximating subspaces are taken to be $H_{u}^{N}=\operatorname{span}\left\{\mathcal{B}_{u_{k}}\right\}_{k=1}^{\mathcal{N}_{u}}, H_{v}^{N}=$ $\operatorname{span}\left\{\mathcal{B}_{v_{k}}\right\}_{k=1}^{\mathcal{N}_{v}}$ and $H_{w}^{N}=\operatorname{span}\left\{\mathcal{B}_{w_{k}}\right\}_{k=1}^{\mathcal{N}_{w}}$. It should be noted that $H^{N}=H_{u}^{N} \times H_{v}^{N} \times H_{w}^{N} \subset V$. The approximating system is then determined by restricting the weak form (2.8) to $H^{N}$ with basis functions used as test functions. This is equivalent to orthogonalizing the residual with respect to elements from $H^{N}$.

\subsection{Matrix System}

To form the resulting matrix system, the generalized Fourier coefficients for the three expansions (4.1) are first consolidated in the vectors

$$
\mathcal{U}^{\mathcal{N}_{u}}(t)=\left[\begin{array}{c}
u_{1}(t) \\
\vdots \\
u_{\mathcal{N}_{u}}(t)
\end{array}\right], \quad \mathcal{V}^{\mathcal{N}_{v}}(t)=\left[\begin{array}{c}
v_{1}(t) \\
\vdots \\
v_{\mathcal{N}_{v}}(t)
\end{array}\right], \quad \mathcal{W}^{\mathcal{N}_{w}}(t)=\left[\begin{array}{c}
w_{1}(t) \\
\vdots \\
w_{\mathcal{N}_{u}}(t)
\end{array}\right]
$$

The full set of coefficients is then represented as $\vartheta^{\mathcal{N}}(t)=\left[\mathcal{U}(t)^{\mathcal{N}_{u}}, \mathcal{V}^{\mathcal{N}_{v}}(t), \mathcal{W}^{\mathcal{N}_{w}}(t)\right]^{T}$, where $\mathcal{N}=\mathcal{N}_{u}+\mathcal{N}_{v}+\mathcal{N}_{w}$.

The mass, stiffness and damping matrices as well as the forcing vector for the full system have the form

$$
\begin{aligned}
& M^{\mathcal{N}}=\left[\begin{array}{c|c|c}
U_{M} & & \\
\hline & V_{M} & \\
\hline & & W_{M}
\end{array}\right], \\
& K_{E}^{\mathcal{N}}=\left[\begin{array}{c|c|c}
U_{11}+U_{12} & V_{11}+V_{12} & W_{11} \\
\hline U_{21}+U_{22} & V_{21}+V_{22} & W_{21} \\
\hline U_{31} & V_{31} & \sum_{k=1}^{6} W_{3 k}
\end{array}\right], \\
& K_{c_{D}}^{\mathcal{N}}=\left[\begin{array}{c|c|c}
\tilde{U}_{11}+\tilde{U}_{12} & \tilde{V}_{11}+\tilde{V}_{12} & \tilde{W}_{11} \\
\hline \tilde{U}_{21}+\tilde{U}_{22} & \tilde{V}_{21}+\tilde{V}_{22} & \tilde{W}_{21} \\
\hline \tilde{U}_{31} & \tilde{V}_{31} & \sum_{k=1}^{6} \tilde{W}_{3 k}
\end{array}\right]
\end{aligned}
$$

and

$$
\hat{F}^{\mathcal{N}}(t)=\left[\begin{array}{c}
F_{u} \\
F_{v} \\
F_{w}
\end{array}\right]
$$

The various submatrices contain individual components which arise when the weak form (2.8) is restricted to $H^{N}$. For example, the approximation of the mass and stiffness components in the longitudinal equation of (2.8) yields 
(i) $\left[U_{M}\right]_{j, k}=\int_{0}^{2 \pi} \int_{0}^{\ell}\left[\rho h+2 \sum_{i=1}^{s} \rho_{p e} h_{p e} \chi_{p e_{i}}\right] R \mathcal{B}_{u_{k}} \mathcal{B}_{u j} d x d \theta$

(ii) $\left[U_{11}\right]_{j, k}=\int_{0}^{2 \pi} \int_{0}^{l}\left[\frac{E h R}{1-\nu^{2}}+\sum_{i=1}^{s} \frac{2 E_{p e} h_{p e} R}{1-\nu_{p e}^{2}} \chi_{p e_{i}}\right] \frac{\partial \mathcal{B}_{u_{k}}}{\partial x} \frac{\partial \mathcal{B}_{u_{j}}}{\partial x} d x d \theta$

(iii)

$$
\left[V_{11}\right]_{j, k}=\int_{0}^{2 \pi} \int_{0}^{\ell}\left[\frac{E h \nu}{1-\nu^{2}}+\sum_{i=1}^{s} \frac{2 E_{p e} h_{p e} \nu_{p e}}{1-\nu_{p e}^{2}} \chi_{p e_{i}}\right] \frac{\partial \mathcal{B}_{v_{k}}}{\partial \theta} \frac{\partial \mathcal{B}_{u_{j}}}{\partial x} d x d \theta
$$

(iv) $\left[W_{11}\right]_{j, k}=\int_{0}^{2 \pi} \int_{0}^{\ell}\left[\frac{E h \nu}{1-\nu^{2}}+\sum_{i=1}^{s} \frac{2 E_{p e} h_{p e} \nu_{p e}}{1-\nu_{p e}^{2}} \chi_{p e_{i}}\right] \mathcal{B}_{w_{k}} \frac{\partial \mathcal{B}_{u_{j}}}{\partial x} d x d \theta$

(v) $\left[V_{12}\right]_{j, k}=\int_{0}^{2 \pi} \int_{0}^{\ell}\left[\frac{E h}{2(1+\nu)}+\sum_{i=1}^{s} \frac{E_{p e} h_{p e}}{1+\nu_{p e}} \chi_{p e_{i}}\right] \frac{\partial \mathcal{B}_{v_{k}}}{\partial x} \frac{\partial \mathcal{B}_{u_{j}}}{\partial \theta} d x d \theta$

(vi)

$$
\left[U_{12}\right]_{j, k}=\int_{0}^{2 \pi} \int_{0}^{\ell}\left[\frac{E h}{2 R(1+\nu)}+\sum_{i=1}^{s} \frac{E_{p e} h_{p e}}{R\left(1+\nu_{p e}\right)} \chi_{p e_{i}}\right] \frac{\partial \mathcal{B}_{u_{k}}}{\partial \theta} \frac{\partial \mathcal{B}_{u_{j}}}{\partial \theta} d x d \theta
$$

(vii) $\left[F_{u}\right]_{j}=\int_{0}^{2 \pi} \int_{0}^{\ell}\left\{R \hat{q}_{x} \mathcal{B}_{u,}+R \sum_{i=1}^{s}\left(N_{x}\right)_{p e_{i}} \frac{\partial \mathcal{B}_{u_{j}}}{\partial x}\right\} d x d \theta$

with similar expressions for the submatrices $\tilde{U}_{11}, \tilde{V}_{11}, \tilde{W}_{11}, \tilde{V}_{12}$ and $\tilde{U}_{12}$ which contain the internal damping contributions. The submatrices for the approximated circumferential and transverse equation are constructed analogously with details given in [16]. It should be noted that due to the presence of the characteristic functions in the force and moment resultants, the domains of integration for the patch contributions are restricted to those regions covered by patches. This is consistent with the fact that material and geometric changes, as well as patch inputs, occur only in those regions.

In first-order form, the system has the form

$$
\begin{aligned}
& {\left[\begin{array}{cc}
K_{E}^{\mathcal{N}} & 0 \\
0 & M^{\mathcal{N}}
\end{array}\right]\left[\begin{array}{l}
\dot{\vartheta}^{\mathcal{N}}(t) \\
\ddot{\vartheta}^{\mathcal{N}}(t)
\end{array}\right]=\left[\begin{array}{cc}
0 & K_{E}^{\mathcal{N}} \\
-K_{E}^{\mathcal{N}} & -K_{c_{D}}^{\mathcal{N}}
\end{array}\right]\left[\begin{array}{l}
\vartheta^{\mathcal{N}}(t) \\
\dot{\vartheta}^{\mathcal{N}}(t)
\end{array}\right]+\left[\begin{array}{c}
0 \\
\hat{F}^{\mathcal{N}}(t)
\end{array}\right]} \\
& {\left[\begin{array}{cc}
K_{E}^{\mathcal{N}} & 0 \\
0 & M^{\mathcal{N}}
\end{array}\right]\left[\begin{array}{l}
\vartheta^{\mathcal{N}}(0) \\
\dot{\vartheta}^{\mathcal{N}}(0)
\end{array}\right]=\left[\begin{array}{l}
g_{1}^{\mathcal{N}} \\
g_{2}^{\mathcal{N}}
\end{array}\right] .}
\end{aligned}
$$

Multiplication of the inverted mass matrix yields a Cauchy equation of the form

$$
\begin{aligned}
& \dot{y}^{N}(t)=A^{N} y^{N}(t)+g^{N}(t) \\
& y^{N}(0)=y_{0}^{N},
\end{aligned}
$$


where $y^{N} \in R^{2 \mathcal{N}}=\left[\vartheta^{\mathcal{N}}(t), \dot{\vartheta}^{\mathcal{N}}(t)\right]^{T}$. This yields a form which is suitable for simulations, parameter estimation and control applications. Note that the system can also be adapted to alternative boundary conditions through modifications of the first and last basis functions. Flexibility in this regard is also a hallmark of the method.

Finally, we point out that approximation of the eigenvalue problem (3.2) using the FourierGalerkin method yields the generalized matrix eigenvalue problem

$$
K_{E}^{\mathcal{N}} \vartheta^{\mathcal{N}}=\omega^{2} M^{\mathcal{N}} \vartheta^{\mathcal{N}}
$$

where $K_{E}^{\mathcal{N}}$ and $M^{\mathcal{N}}$ are defined in (4.9). As illustrated in the examples of Section 5.1, (4.13) can be used to approximate the frequencies and modes for undamped shells with various internal characteristics (due to actuators and sensors) and boundary conditions.

\section{$5 \quad$ Examples}

To illustrate various facets of the Fourier-Galerkin method, two types of examples are presented. In the first set of examples, approximation of frequencies and modes is considered for simply-supported and fixed-edge shells. This is accomplished by forming the mass and stiffness matrices and solving the generalized matrix eigenvalue problem (4.13). This serves two purposes. It illustrates the accuracy of the method in approximating the mass and stiffness components in the system and demonstrates the method as an effective technique for obtaining modal information when analytic or experimental values are unavailable.

The second set of examples illustrates the approximation of static and dynamic shell responses to various forces. The initial steady state example illustrates that the expected $\mathcal{O}\left(h_{x}^{2}\right)$ and $\mathcal{O}\left(h_{x}^{4}\right)$ convergence rates are obtained when approximating a static shell response (this example involves the solution of a matrix system with coefficient matrix $K_{E}^{\mathcal{N}}$ and vector $\left.\hat{F}^{\mathcal{N}}\right)$. Analogous dynamic examples demonstrate that the same accuracy is obtained when the method is used to obtain the time-dependent system (4.12) which is then marched in time. The latter example also incorporates the internal Kelvin-Voigt damping. The dynamics of the system in response to patch inputs are considered in the final example. Natural frequencies are compared to those obtained by solving the generalized matrix eigenvalue problem to illustrate consistency among techniques.

In these examples, the following shell characteristics were used: thickness $h=.01 \mathrm{in}$, length $\ell=12 \mathrm{in}$, radius $R=3.0 \mathrm{in}$, mass density $\rho=.283 \mathrm{lb} / \mathrm{in}^{3}$, Young's modulus $E=3.0 \times$ $10^{7} \mathrm{lb} / \mathrm{in}^{2}$, Poisson ratio $\nu=0.3$ and Kelvin-Voigt damping coefficient $c_{D}=15.09936 \mathrm{lb} \cdot \mathrm{in} \cdot \mathrm{s}^{2}$. These values were chosen so as to permit comparison with Donnell-Mushtari shell results in [24, pp. 307-310]. We emphasize that the method is not restricted to such dimensions or ratios, however, and analogous convergence results have been obtained with a wide range of parameters.

\subsection{Modal Examples: Simply-Supported Shell}

In Section 3, separation of variables was used to obtain analytic expressions for modes and natural frequencies of shell models with simply-supported boundary conditions. Here we compare the approximate solutions obtained by solving the matrix eigenvalue problem (4.13) with 
the analytic values. This provides a means of testing the accuracy of the method before using it to approximate in settings in which analytic solutions are unavailable. To illustrate, we consider the cases of purely axisymmetric modes $(m=0, n \geq 1)$, purely extensional modes $(m \geq 1, n=0)$ and general modes $(m \geq 1, n \geq 1)$.

\subsection{1 $m=0, n \geq 1$ Axisymmetric Modes}

Analytic frequencies for the axisymmetric case can be calculated via (3.6) with $m=0$ while corresponding modes can be determined from (3.3) or (3.7) (see [16] for details). Corresponding approximate frequencies, obtained by solving (4.13) with Fourier limit $M=0$, are compared with analytic values in Table 3. As noted in Section 3 , three frequencies are obtained for each configuration of $1 / 2$ wavelength (fixed values of $n$ ), and this is reflected in the analytic and approximate results in Table 3 . Two of the frequencies correspond to coupled axial/radial modes while the remaining one corresponds to an uncoupled torsional (circumferential) mode. The frequencies of the torsional modes are boxed to facilitate comparison between the corresponding functional modal approximations.

The cubic spline approximates in Table 3 were obtained using $N=8$ basis functions whereas $N=32$ linear splines were used to obtain the corresponding results in columns 8-10. It is noted that accurate frequency approximates are obtained with the cubic splines with a maximum relative error (over the reported results) of $0.3 \%$ occurring for the frequency $2255.52 \mathrm{~Hz}$. Due to the limited accuracy of the linear splines, significantly more basis functions must be used to obtain accurate approximations, and a $1.25 \%$ error at $2255.52 \mathrm{~Hz}$ remains, even with $N=32$ functions.

For $n=1$, cross sections at $\theta=0$ of corresponding modes approximated using $M=0, N=$ 16 cubic splines are plotted in Figure 2. The coupling between the axial/radial components is readily verified for the $406.8 \mathrm{~Hz}$ and $603.83 \mathrm{~Hz}$ modes while the plot of the $266.05 \mathrm{~Hz}$ mode illustrates that for $M=0$, the torsional mode is uncoupled (examples in Section 5.1.3 illustrate that all three modes are coupled for $M \geq 1$ ).

It should be noted that for each $n$, all three frequencies and modes are automatically yielded by the approximation method whereas (3.3) and (3.7) must be employed to obtain analytic expressions for the axial/radial and torsional modes, respectively. In this aspect, the approximation method facilitates the calculation of a complete modal set.

\begin{tabular}{l||rrr||rrr||rrr}
\hline$n$ & \multicolumn{3}{|c||}{ Analytic Frequencies } & \multicolumn{3}{c||}{ Cubic Spline Approx } & \multicolumn{3}{c}{ Linear Spline Approx } \\
\hline \hline 1 & 266.05 & 406.80 & 603.83 & 266.06 & 406.80 & 603.83 & 266.19 & 406.99 & 603.87 \\
2 & 531.53 & 532.11 & 924.29 & 531.53 & 532.11 & 924.29 & 531.71 & 533.18 & 925.95 \\
3 & 541.03 & 798.16 & 1362.11 & 541.03 & 798.22 & 1362.19 & 541.25 & 801.79 & 1368.07 \\
4 & 543.53 & 1064.22 & 1807.86 & 543.58 & 1064.82 & 1808.81 & 543.85 & 1072.81 & 1822.21 \\
5 & 544.60 & 1330.27 & 2255.52 & 544.85 & 1333.95 & 2262.44 & 545.06 & 1347.10 & 2283.78 \\
\hline
\end{tabular}

Table 3. Analytic frequencies and approximate values obtained using $N=8$ cubic splines and $N=32$ linear splines. Frequencies of the torsional modes are boxed. 

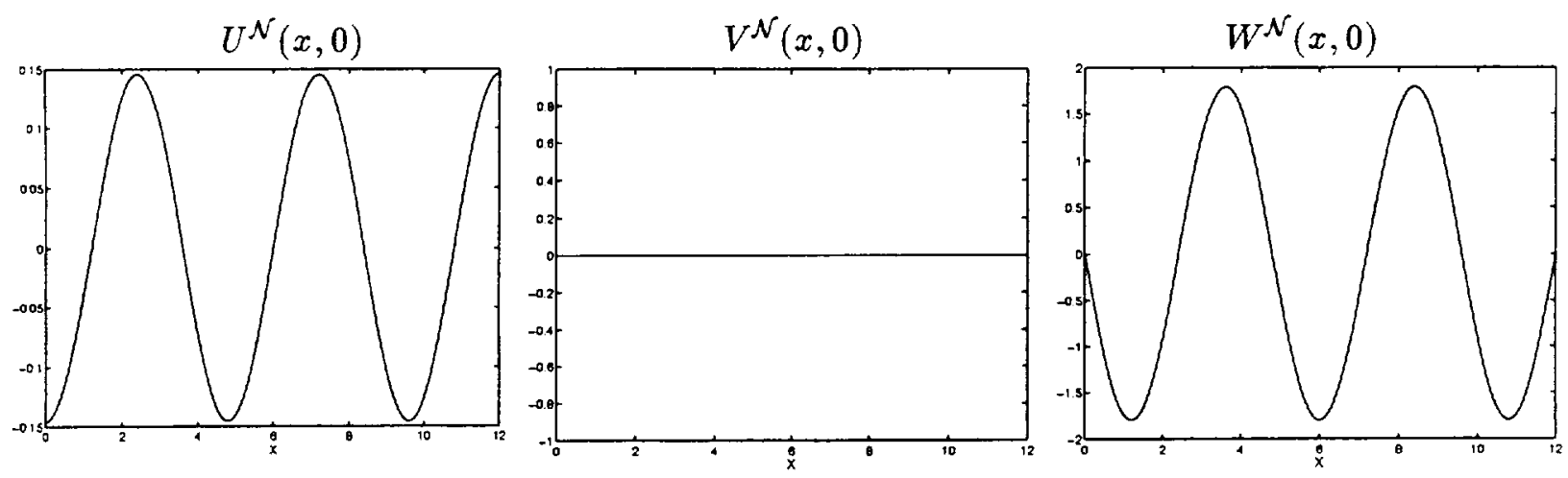

$544.60 \mathrm{~Hz}$
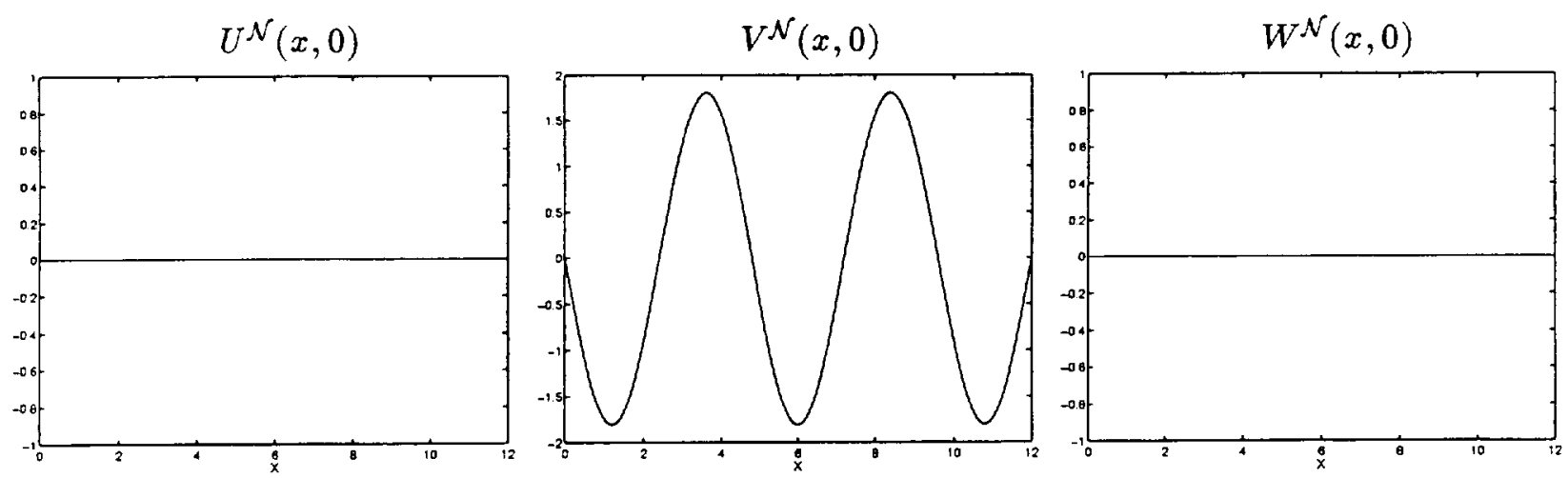

$1330.27 \mathrm{~Hz}$
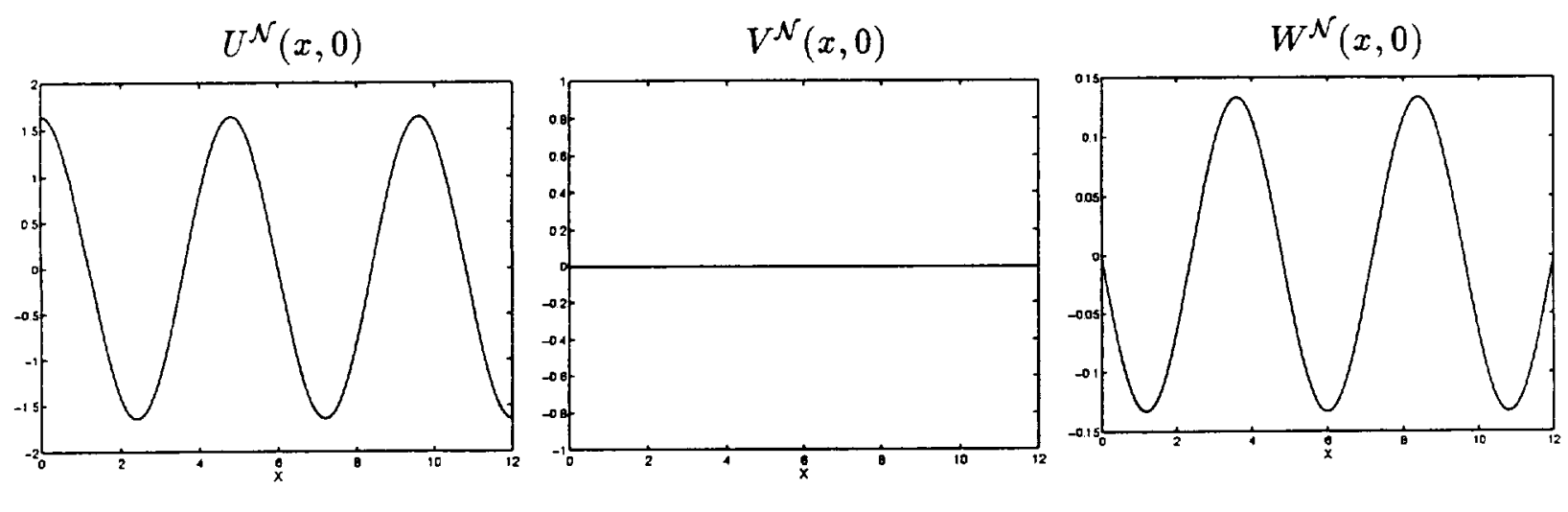

$2255.52 \mathrm{~Hz}$

Figure 2. Approximate axisymmetric modes $U^{\mathcal{N}}(x, 0), V^{\mathcal{N}}(x, 0)$ and $W^{\mathcal{N}}(x, 0)$ corresponding to the $544.60,1330.27$ and $2255.52 \mathrm{~Hz}$ natural frequencies. 


\subsection{2 $m \geq 1, n=0$ Purely Extensional Modes}

A second case in which analytic expressions for the shell frequencies and modes are easily expressed occurs when purely extensional modes are present. The first three analytic frequencies given by (3.6) are compared in Table 4 with approximate values obtained with $N=8$ cubic splines. Due to the accuracy of the method, the approximate frequencies agree with analytic values to within at least two decimal places in all three cases. Corresponding modal plots are given in Figure 3. The numerical plots in the left column illustrate the two approximate solutions, for each frequency, obtained by solving the matrix eigenvalue problem (4.13) (again, the full solution set is automatically obtained by the approximation method). The corresponding analytic modes given by (3.3) and (3.7) are depicted in the right column of the figure. With $n=0$, it is seen from (3.3) and (3.7) that the analytic expressions for the longitudinal modes are $U(x, \theta)=A_{1} \cos (m \theta)$ and $U(x, \theta)=A_{2} \sin (m \theta)$ with zero displacement expected in the circumferential and transverse components. This longitudinal behavior is noted for both the analytic and approximate modes in Figure 3.

\begin{tabular}{l|cc}
\hline & Analtyic (Hz) & Galerkin (Hz) \\
\hline$m=1, n=0$ & 338.75 & 338.75 \\
$m=2, n=0$ & 677.50 & 677.50 \\
$m=3, n=0$ & 1016.25 & 1016.25 \\
\hline
\end{tabular}

Table 4. Analytic and approximate frequencies for purely extensional modes obtained using $N=8$ cubic splines.

\subsection{3 $m \geq 1, n \geq 1$ General Shell Modes}

For the general case with $m \geq 1, n \geq 1$, the axial, radial and torsional modes are all coupled with analytic values for the natural frequencies determined by (3.6) where $\Omega_{m n j}^{2}$ is one of the three solutions to the cubic equation (3.5). These analytic values are compared in Table 5 with approximate frequencies obtained using the cubic spline basis with $N=8, M=3$. For comparison sake, frequencies of the axisymmetric $(m=0)$ and purely extensional $(n=0)$ are also included in this table. The accuracy of the cubic spline discretization leads to approximate frequencies having relative errors less than $0.5 \%$ for the reported values (the largest relative error for the reported values occurs in the second frequency obtained with $m=3, n=5$ ). The convergence of the method and accuracy obtained using the cubic splines is further illustrated by the frequency results in Table 6 where approximates obtained with $N=8$ and $N=16$ are compared. A check of the relative errors shows that the method is converging more quickly than the expected $\mathcal{O}\left(h_{x}^{4}\right)$ rate.

The modes for this general case are again obtained from (3.3) and (3.7). The coupling between the axial, radial and torsional modes can be seen in Figure 4 where the $873.61 \mathrm{~Hz}$ mode is plotted along the axial line $\ell=\{(x, \theta) \mid \theta=\pi / 4,0 \leq x \leq 12\}$.

We reiterate that the full set of frequencies and modes can be approximated using the Fourier-Galerkin method by simply solving the matrix eigenvalue problem (4.13). Moreover, this technique can be directly extended to the problem of calculating frequencies and modes for shells with other boundary conditions by suitably modifying the spline basis. This is illustrated in the next section where the modal analysis of a shell with clamped ends is considered. 

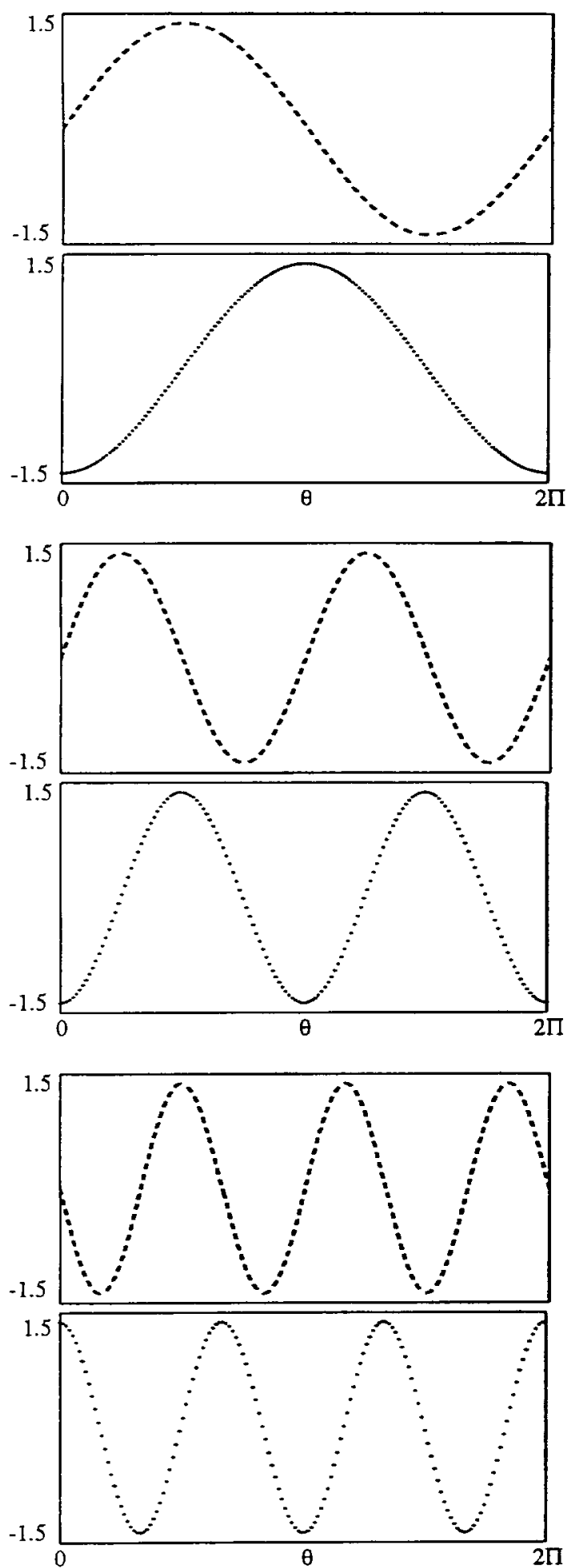
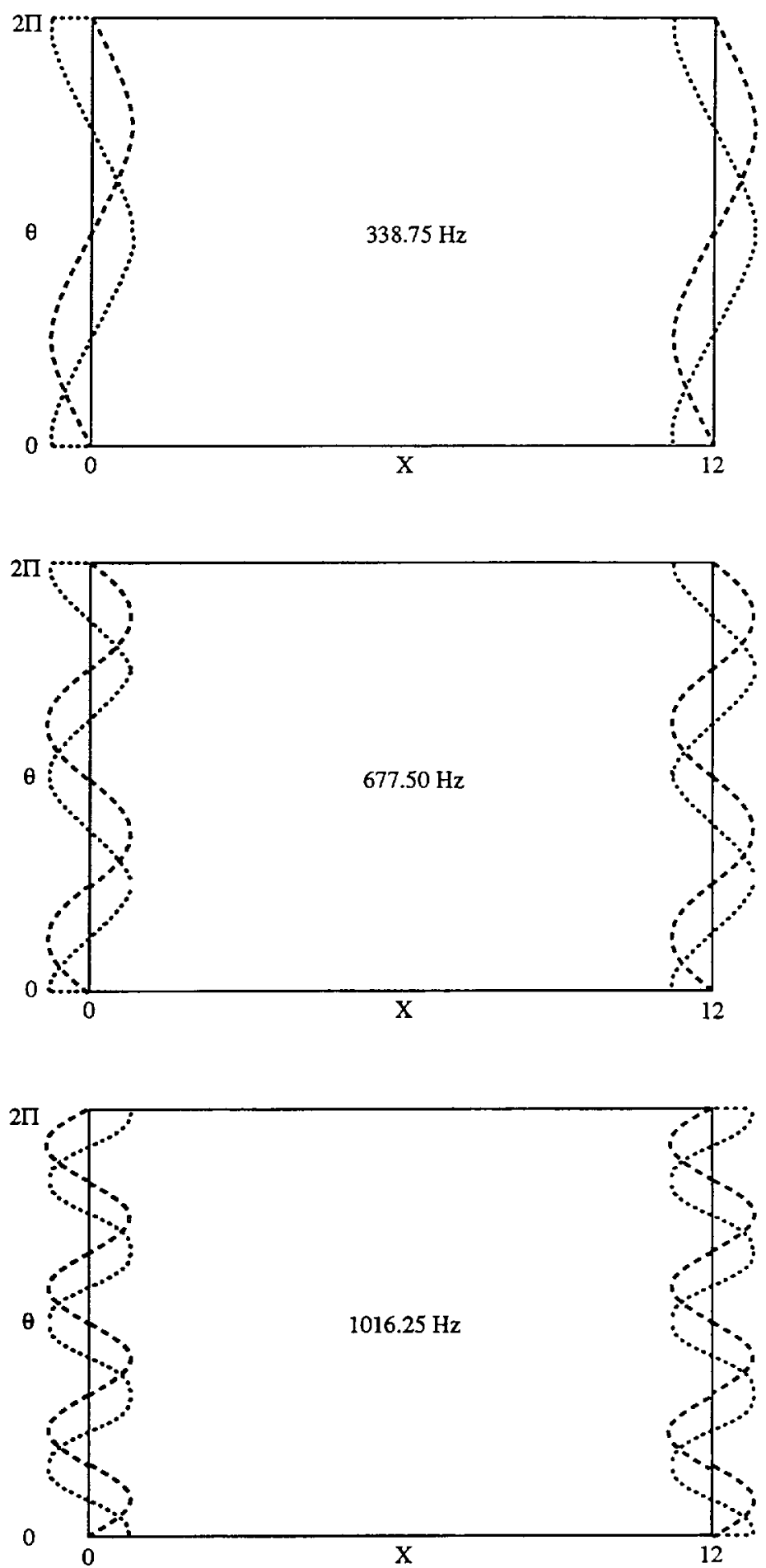

Figure 3. Purely extensional modes in the longitudinal direction. For $N=8$ cubic splines, numerical values of $U^{\mathcal{N}}$ along the curve $\ell=\{(x, \theta) \mid 0 \leq \theta \leq 2 \pi, x=0\}$ are plotted in left column. Corresponding shell modes given by (3.3) and (3.7) with $n=0$ are depicted in right column. 


\begin{tabular}{|c|c|c|c|c|c|c|c|}
\hline \multicolumn{4}{|c|}{ Analytic Frequencies } & \multicolumn{4}{|c|}{ Galerkin Approximates } \\
\hline \multicolumn{4}{|c|}{$m=0$} & \multicolumn{4}{|c|}{$m=0$} \\
\hline$n=1$ & 266.05 & 406.80 & 603.83 & $n=1$ & 266.06 & 406.80 & 603.83 \\
\hline$n=2$ & 531.53 & 532.11 & 924.29 & $n=2$ & 531.53 & 532.11 & 924.29 \\
\hline$n=3$ & 541.03 & 798.16 & 1362.11 & $n=3$ & 541.03 & 798.22 & 1362.19 \\
\hline$n=4$ & 543.53 & 1064.22 & 1807.86 & $n=4$ & 543.58 & 1064.82 & 1808.81 \\
\hline$n=5$ & 544.60 & 1330.27 & 2255.52 & $n=5$ & 544.85 & 1333.95 & 2262. \\
\hline \multicolumn{4}{|c|}{$m=1$} & \multicolumn{4}{|c|}{$m=1$} \\
\hline$n=0$ & \multicolumn{3}{|c|}{338.75} & $n=0$ & \multicolumn{3}{|c|}{338.75} \\
\hline$n=1$ & 147.09 & 508.59 & 873.61 & $n=1$ & 147.09 & 508.59 & 873.61 \\
\hline$n=2$ & 327.96 & 714.55 & 1115.55 & $n=2$ & 327.96 & 714.44 & 1115.55 \\
\hline$n=3$ & 434.81 & 909.78 & 1486.93 & $n=3$ & 434.83 & 909.85 & 1487.00 \\
\hline$n=4$ & 483.84 & 1137.35 & 1900.34 & $n=4$ & 483.92 & 1138.09 & 1901.13 \\
\hline$n=5$ & 507.08 & 1383.62 & 2329.09 & $n=5$ & 507.48 & 1388.30 & 2335.07 \\
\hline \multicolumn{4}{|c|}{$m=2$} & \multicolumn{4}{|c|}{$m=2$} \\
\hline$n=0$ & \multicolumn{3}{|c|}{677.50} & $n=0$ & \multicolumn{3}{|c|}{677.50} \\
\hline$n=1$ & 64.56 & 755.92 & 1340.06 & $n=1$ & 64.56 & 755.92 & 1340.06 \\
\hline$n=2$ & 187.34 & 917.53 & 1521.01 & $n=2$ & 187.35 & 917.53 & 1521.01 \\
\hline$n=3$ & 296.26 & 1100.21 & 1804.82 & $n=3$ & 296.28 & 1100.29 & 1804.85 \\
\hline$n=4$ & 373.46 & 1300.70 & 2153.02 & $n=4$ & 373.61 & 1301.67 & 2153.52 \\
\hline$n=5$ & 423.98 & 1519.53 & 2536.80 & $n=5$ & 424.54 & 1521.01 & 2540.73 \\
\hline \multicolumn{4}{|c|}{$m=3$} & \multicolumn{4}{|c|}{$m=3$} \\
\hline$n=0$ & \multicolumn{3}{|c|}{1016.25} & $n=0$ & \multicolumn{3}{|c|}{1016.25} \\
\hline$n=1$ & 33.48 & 1061.88 & 1858.96 & $n=1$ & 33.48 & 1061.88 & 1858.96 \\
\hline$n=2$ & 111.09 & 1177.72 & 2001.04 & $n=2$ & 111.09 & 1177.73 & 2001.04 \\
\hline$n=3$ & 198.51 & 1332.12 & 2225.92 & $n=3$ & 198.54 & 1332.21 & 2225.94 \\
\hline$n=4$ & 275.56 & 1509.99 & 2514.74 & $n=4$ & 275.76 & 1511.08 & 2515.01 \\
\hline$n=5$ & 336.44 & 1706.31 & 2848.26 & $n=5$ & 337.29 & 1714.42 & 2850.19 \\
\hline
\end{tabular}

Table 5. Frequencies approximated with $M=3$ and $N=8$ cubic basis functions. 


\begin{tabular}{l|lll}
\hline \multicolumn{5}{c}{$m=0, n=5$} \\
\hline$N=8$ & 544.85 & 1333.95 & 2262.44 \\
$N=16$ & 544.60 & 1330.30 & 2255.57 \\
Analytic & 544.60 & 1330.27 & 2255.52 \\
\hline \hline \multicolumn{4}{c}{$m=1, n=5$} \\
\hline$N=8$ & 507.48 & 1388.30 & 2335.07 \\
$N=16$ & 507.08 & 1383.66 & 2329.14 \\
Analytic & 507.08 & 1383.62 & 2329.09 \\
\hline \hline \multicolumn{5}{c}{$m=2, n=5$} \\
\hline$N=8$ & 424.54 & 1521.01 & 2540.73 \\
$N=16$ & 423.99 & 1519.56 & 2536.84 \\
Analytic & 423.98 & 1519.53 & 2536.80 \\
\hline \hline \multicolumn{5}{c}{$m=3, n=5$} \\
\hline$N=8$ & 337.27 & 1714.42 & 2850.19 \\
$N=16$ & 336.45 & 1706.35 & 2848.28 \\
Analytic & 336.44 & 1706.31 & 2848.26 \\
\hline
\end{tabular}

Table 6. Frequencies approximated with $M=3$ and $N=8,16$ cubic basis functions.
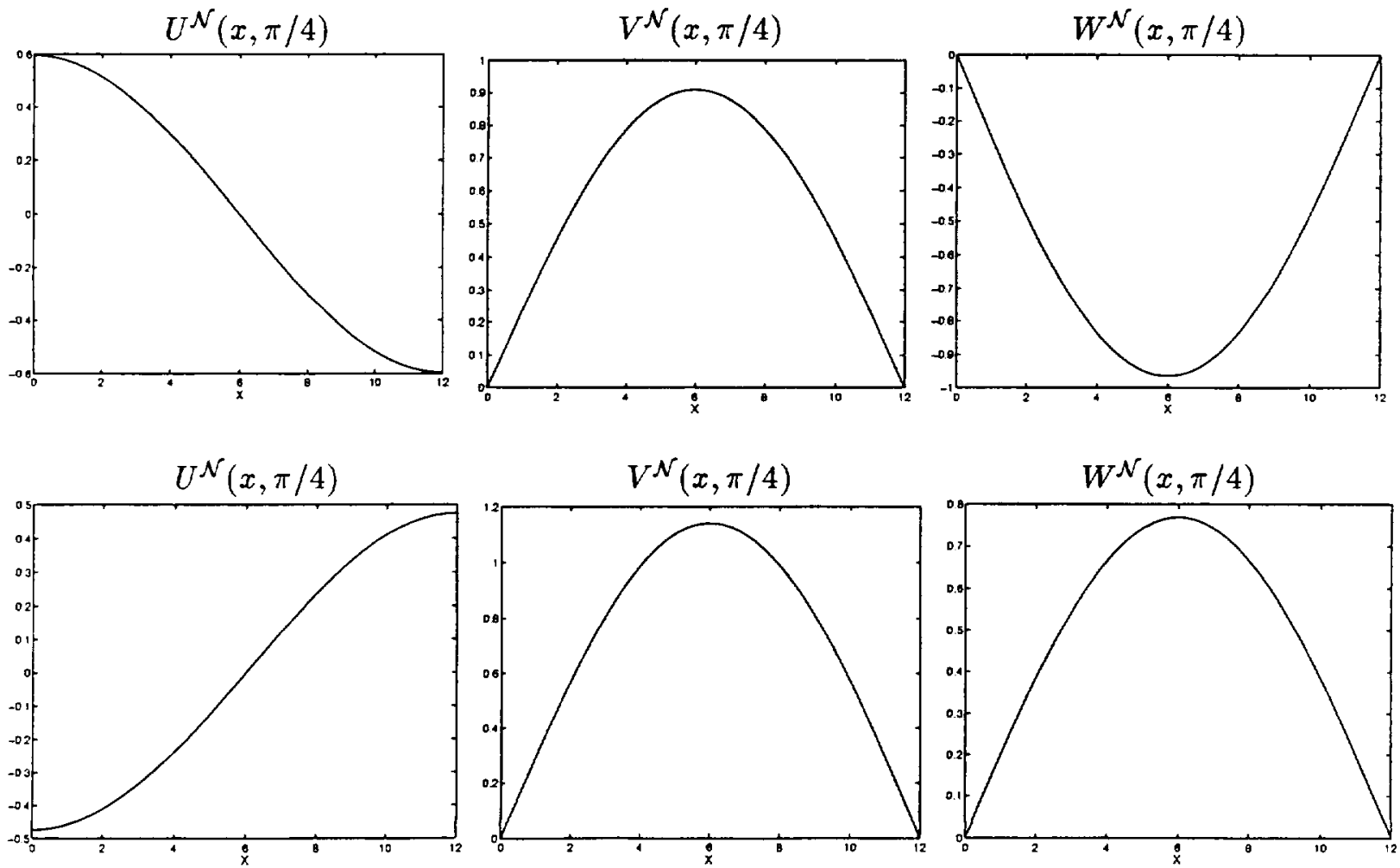

Figure 4. Approximate $873.61 \mathrm{~Hz}$ modes along the line $\ell=\{(x, \theta) \mid \theta=\pi / 4,0 \leq x \leq 12\}$. 


\subsection{Modal Example: Fixed-Edge Shell}

The lack of analytic expressions for the frequencies of a clamped-end shell necessitates their numerical approximation. Like the simply supported case, the frequencies are approximated by solving the matrix eigenvalue problem (4.13) with basis functions constructed so as to satisfy the fixed-edge boundary conditions (2.6). Axisymmetric frequencies obtained using a Fourier limit of $M=0$ and $x$-axis basis number of $N=16$ are summarized in Table 7. A comparison with the axisymmetric frequencies of the simply supported shell (see Table 3) shows that only the torsional frequencies match (the torsional frequencies are boxed to facilitate comparison).

\begin{tabular}{|lll|}
\hline \multicolumn{3}{|c|}{ Approximate Frequencies } \\
\hline \hline 266.05 & 410.10 & 532.11 \\
533.22 & 541.76 & 543.99 \\
544.96 & 545.56 & 546.07 \\
546.69 & 547.63 & 549.25 \\
552.18 & 557.39 & 565.57 \\
571.17 & 575.20 & 599.46 \\
\hline 798.16 & 921.82 & 1064.22 \\
\hline 1330.30 & 1360.78 & 1596.45 \\
1806.93 & 1862.80 & 2129.72 \\
2254.85 & 2397.95 & 2669.00 \\
2703.58 & 2945.38 & 3153.02 \\
3230.49 & 3527.16 & 3603.61 \\
3833.08 & 4056.54 & 4119.08 \\
4514.29 & 4981.08 & 5167.03 \\
5172.22 & 5462.62 & 5963.62 \\
6480.17 & 6962.94 & 8734.17 \\
8742.91 & & \\
\hline \hline
\end{tabular}

Table 7. Cubic spline approximates of axisymmetric frequencies obtained with $M=0$ and $N=16$ for the clamped-end shell.

While the torsional modes for the fixed-edge shell match those for a simply-supported shell when $m=0$ (see [16]), the general modes for the two shells differ due to boundary conditions. To illustrate, fixed-edge and simply-supported shell modes having similar frequencies and wavenumbers are compared in Figure 5. Specifically, the $545.56 \mathrm{~Hz}$ mode for 
the clamped-edge shell is compared with the corresponding $545.66 \mathrm{~Hz}$ mode for the simplysupported shell. The plots show that while the qualitative mode shapes are somewhat similar for the two boundary conditions, the clamped-edge shell does not exhibit the trigonometric modal relations which characterize the simply-supported shell. This illustrates the necessity of obtaining approximations consistent with the physical application if employing modal expansions for simulations, damage detection or control.
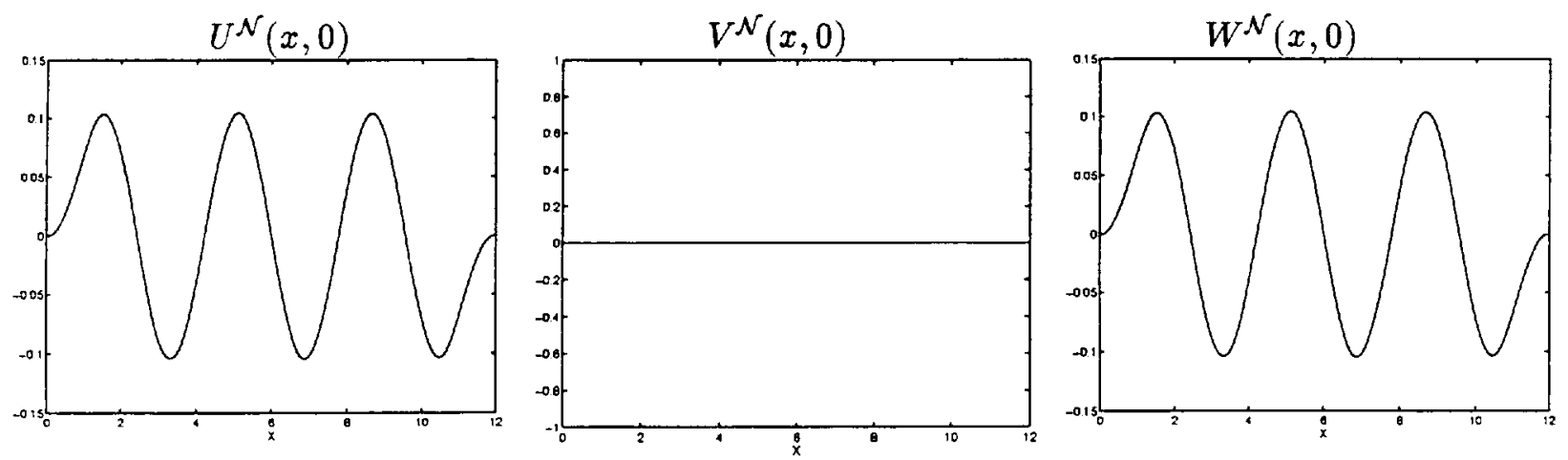

$545.56 \mathrm{~Hz}$, Fixed Edge
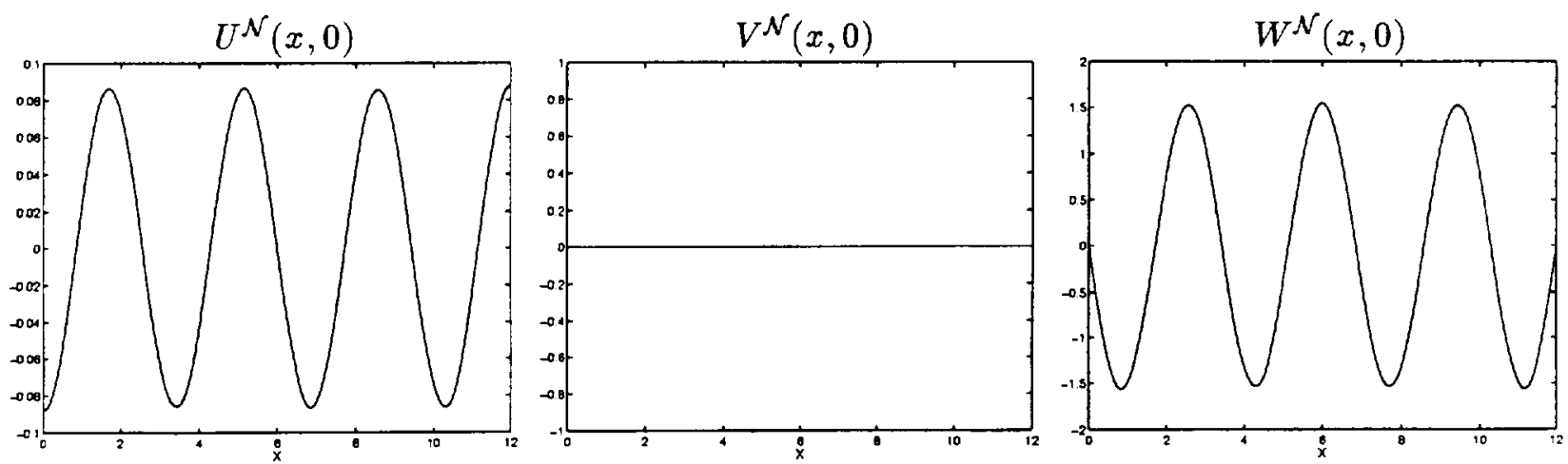

$545.66 \mathrm{~Hz}$, Simply Supported

Figure 5. Axisymmetric modes $U^{\mathcal{N}}(x, 0), V^{\mathcal{N}}(x, 0)$ and $W^{\mathcal{N}}(x, 0)$ corresponding to the fixededge $545.56 \mathrm{~Hz}$ frequency and simply supported $545.66 \mathrm{~Hz}$ frequency.

\subsection{Forced Shell Examples}

A second means of testing the capabilities of the approximation method is through the consideration of steady state and time-dependent shell responses to given force and moment inputs. In the first examples of this section, known true solutions are used to calculate corresponding force input. By comparing the resulting approximate solutions with the original true solutions, the expected $\mathcal{O}\left(h_{x}^{2}\right)$ and $\mathcal{O}\left(h_{x}^{4}\right)$ convergence rates for the linear and cubic splines can be verified. In the final example, a triangular input to the patch terms (modeling a voltage to spike to the patches) provides a broadband model response. The resulting frequencies are then compared with those obtained by solving the matrix eigenvalue problem (4.13) to illustrate the consistency among techniques. 


\subsubsection{Steady State Solution - Linear and Cubic Spline Approximations}

In this example, steady state, $\theta$-dependent solutions satisfying the clamped edge boundary condition are considered. The true displacements were taken to be

$$
\begin{aligned}
& u(x, \theta)=\sin (2 \pi x / \ell) \sin (\theta) \\
& v(x, \theta)=\sin (4 \pi x / \ell) \sin (2 \theta) \\
& w(x, \theta)=[\cos (2 \pi x / \ell)-1] \cos (3 \theta),
\end{aligned}
$$

and hence they satisfy the fixed-edge conditions (2.6). Plots of those displacements are given in Figure 6 to illustrate that significant bending deformations are present. The forcing functions were obtained by substituting the true solutions into the strong form (2.1) of the equations and solving for the functions $\hat{q}_{x}, \hat{q}_{\theta}$ and $\hat{q}_{n}$.

Since the forces are time-independent, the coefficients $\vartheta^{\mathcal{N}}=\left[u_{1}, \cdots, u_{\mathcal{N}_{u}}, v_{1}, \cdots, v_{\mathcal{N}_{v}}\right.$, $\left.w_{1}, \cdots, w_{\mathcal{N}_{w}}\right]^{T}$ for the approximate displacements $u^{N}(\theta, x), v^{N}(\theta, x)$ and $w^{N}(\theta, x)$ of $(4.1)$ are obtained by solving the matrix system

$$
K_{E}^{\mathcal{N}} \vartheta^{\mathcal{N}}=\hat{F}^{\mathcal{N}},
$$

where $K_{E}^{\mathcal{N}}$ and $\hat{F}^{\mathcal{N}}$ are defined in (4.9) and (4.10), respectively. The specific construction and dimensions of $K_{E}^{\mathcal{N}}$ and $\hat{F}^{\mathcal{N}}$ depend upon the bases used in forming $u^{N}, v^{N}$ and $w^{N}$ (see Table 1).

When approximating the solutions to (5.1), both the linear and cubic axial basis functions summarized in Table 1 were considered. For both cases, the maximum absolute errors over a uniform $25 \times 25$ grid in $(x, \theta)$ are summarized in Table 8 while ratios of the errors are given in Table 9 (the absolute errors provide the same information as relative errors since the magnitudes of the solutions are on the order of one). For both sets of approximations, equal axial index limits were assumed and are denoted by $N_{u}=N_{v}=N_{w}=N$. Due to the nature of the forcing function, the Fourier limit $M=3$ was sufficient for resolving the circumferential behavior.

The error results in Table 8 illustrate the rapid convergence of the method with the fully cubic spline basis while the ratios in Table 9 demonstrate the respective $\mathcal{O}\left(h_{x}^{4}\right)$ and $\mathcal{O}\left(h_{x}^{2}\right)$ convergence rates of the cubic and linear bases, respectively (the multiplying constant causes the ratios in the linear case to be slightly less than the expected value of four). It should be noted that due to the coupling between the longitudinal, circumferential and transverse displacements in the modeling equations, the accuracy of the transverse approximate is reduced to $\mathcal{O}\left(h_{x}^{2}\right)$ when linear splines were used to approximate $u$ and $v$, even though cubic splines were used to approximate $w$.

The approximate longitudinal displacements obtained with $N=4,8,16$ and 32 linear axial basis functions are plotted in Figure 7. Comparison with the true solution in Figure 6 illustrates that with $N=16$, the approximation method has captured the qualitative behavior of the solution. Corresponding plots of the circumferential and transverse displacements, plotted in Figures 14 and 15, can be compared with the true circumferential and transverse solutions in Figure 6. 


\begin{tabular}{c|c|c|c||c|c|c|c|}
\cline { 2 - 8 } \multicolumn{1}{c|}{} & \multicolumn{2}{c||}{ Fully Cubic Spline Axial Basis } & \multicolumn{4}{c|}{ Mixed Linear/Cubic Axial Basis } \\
\cline { 2 - 8 } & $N=4$ & $N=8$ & $N=16$ & $N=4$ & $N=8$ & $N=16$ & $N=32$ \\
\hline \hline$u$ & $1.663-1$ & $2.003-3$ & $3.936-5$ & $5.010-1$ & $1.266-1$ & $3.277-2$ & $7.751-3$ \\
$v$ & $5.687-1$ & $1.501-2$ & $6.235-4$ & $1.580+0$ & $2.962-1$ & $8.313-2$ & $2.197-2$ \\
$w$ & $3.136-1$ & $4.075-3$ & $5.552-5$ & $1.864+0$ & $4.903-1$ & $1.236-1$ & $3.784-2$ \\
\hline
\end{tabular}

Table 8. Absolute errors in the longitudinal $u$, circumferential $v$, and transverse displacements $w$ with cubic and linear spline axial basis functions.

\begin{tabular}{c|cc||ccc}
\cline { 2 - 6 } & \multicolumn{2}{c||}{ Cubic Basis } & \multicolumn{3}{c}{ Linear/Cubic Basis } \\
\cline { 2 - 6 } & $\frac{N=4}{N=8}$ & $\frac{N=8}{N=16}$ & $\frac{N=4}{N=8}$ & $\frac{N=8}{N=16}$ & $\frac{N=16}{N=32}$ \\
\hline \hline$u$ & 83.052 & 50.888 & 3.956 & 3.863 & 4.228 \\
$v$ & 37.899 & 24.069 & 5.336 & 3.563 & 3.785 \\
$w$ & 76.961 & 73.395 & 3.801 & 3.966 & 3.268 \\
\hline
\end{tabular}

Table 9. Ratios of the absolute errors with linear and cubic spline axial basis functions.
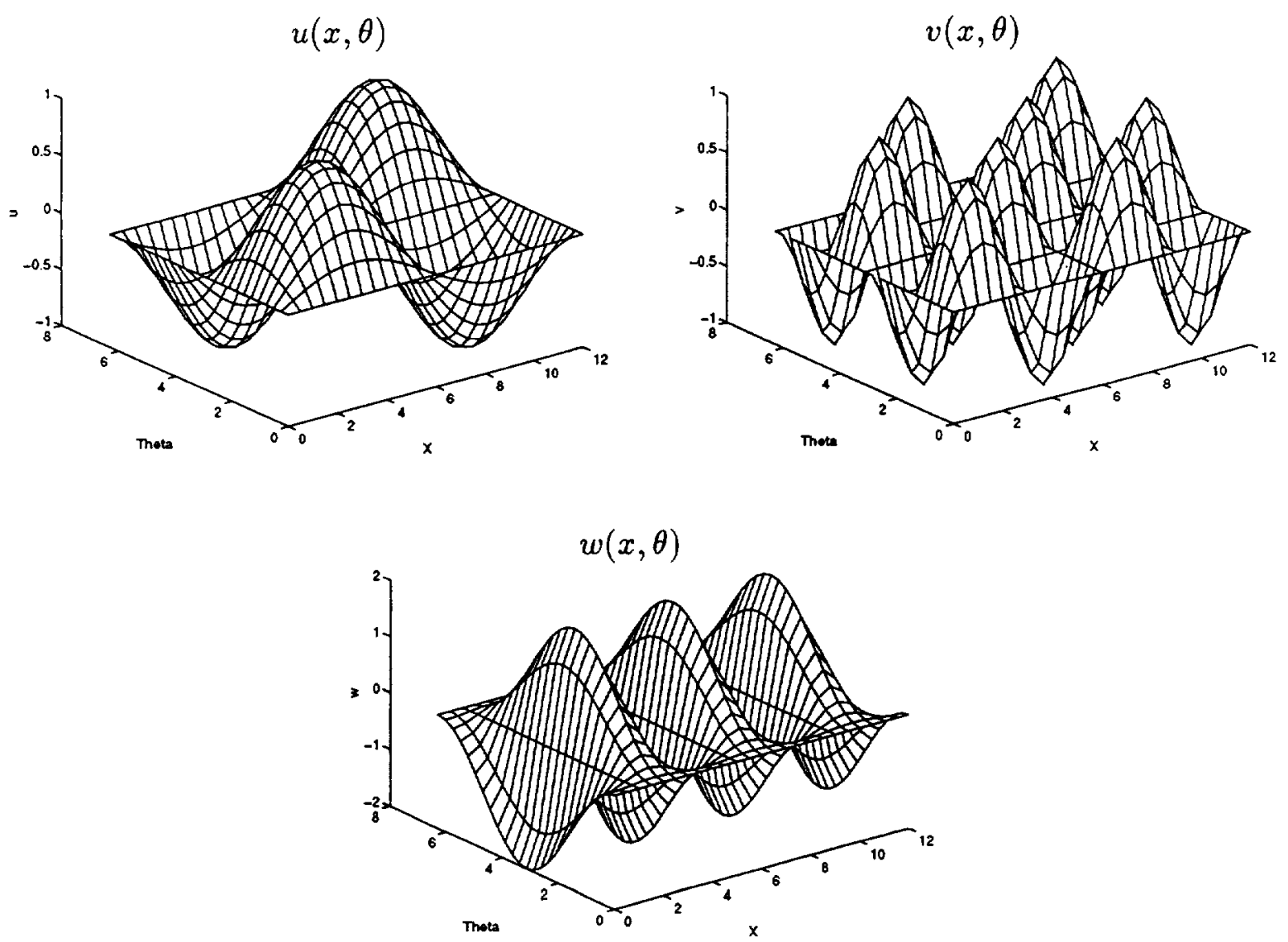

Figure 6. True longitudinal, circumferential and transverse displacements. 

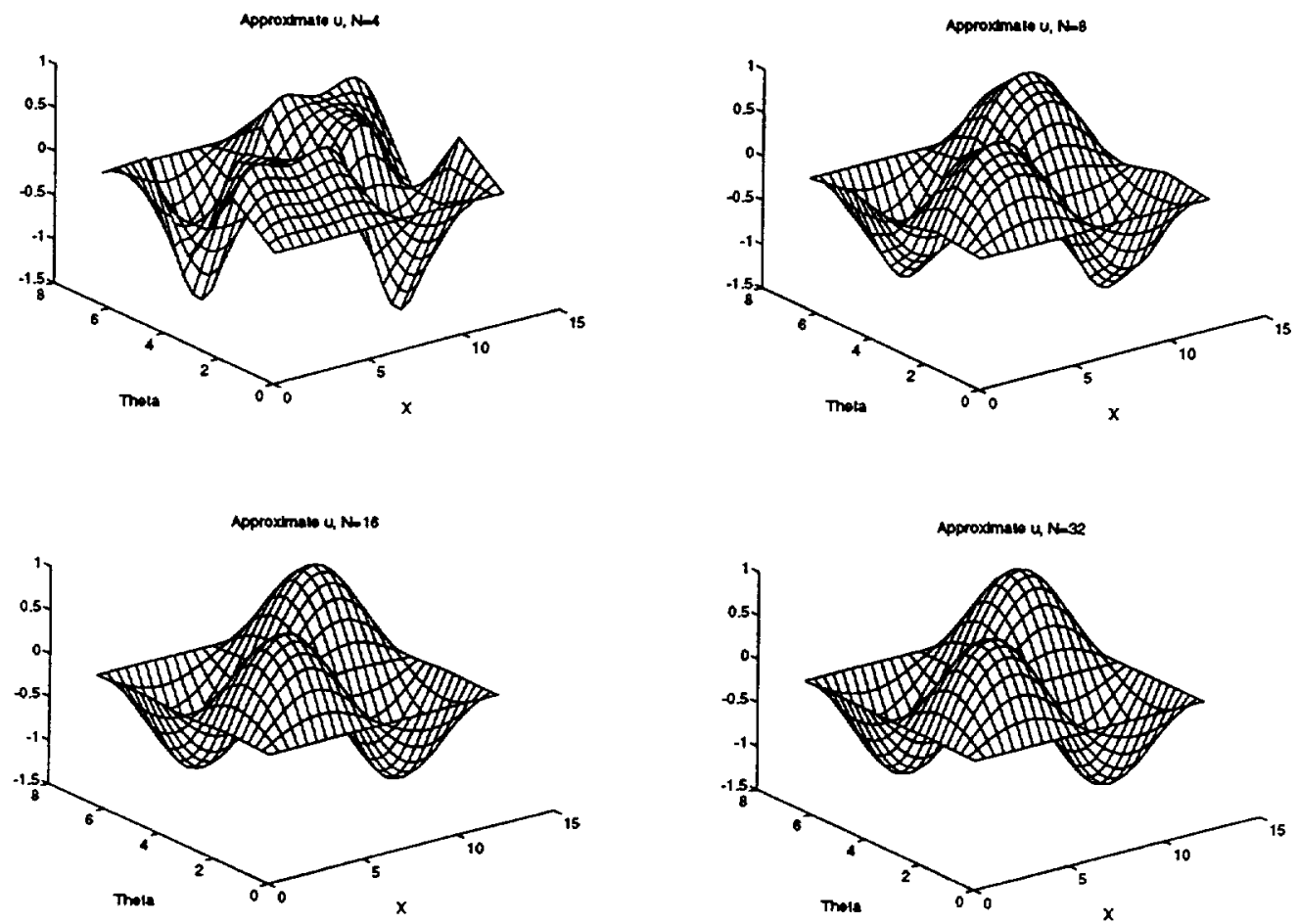

Figure 7. Approximate longitudinal displacement $u$ with $N=4,8,16,32$.
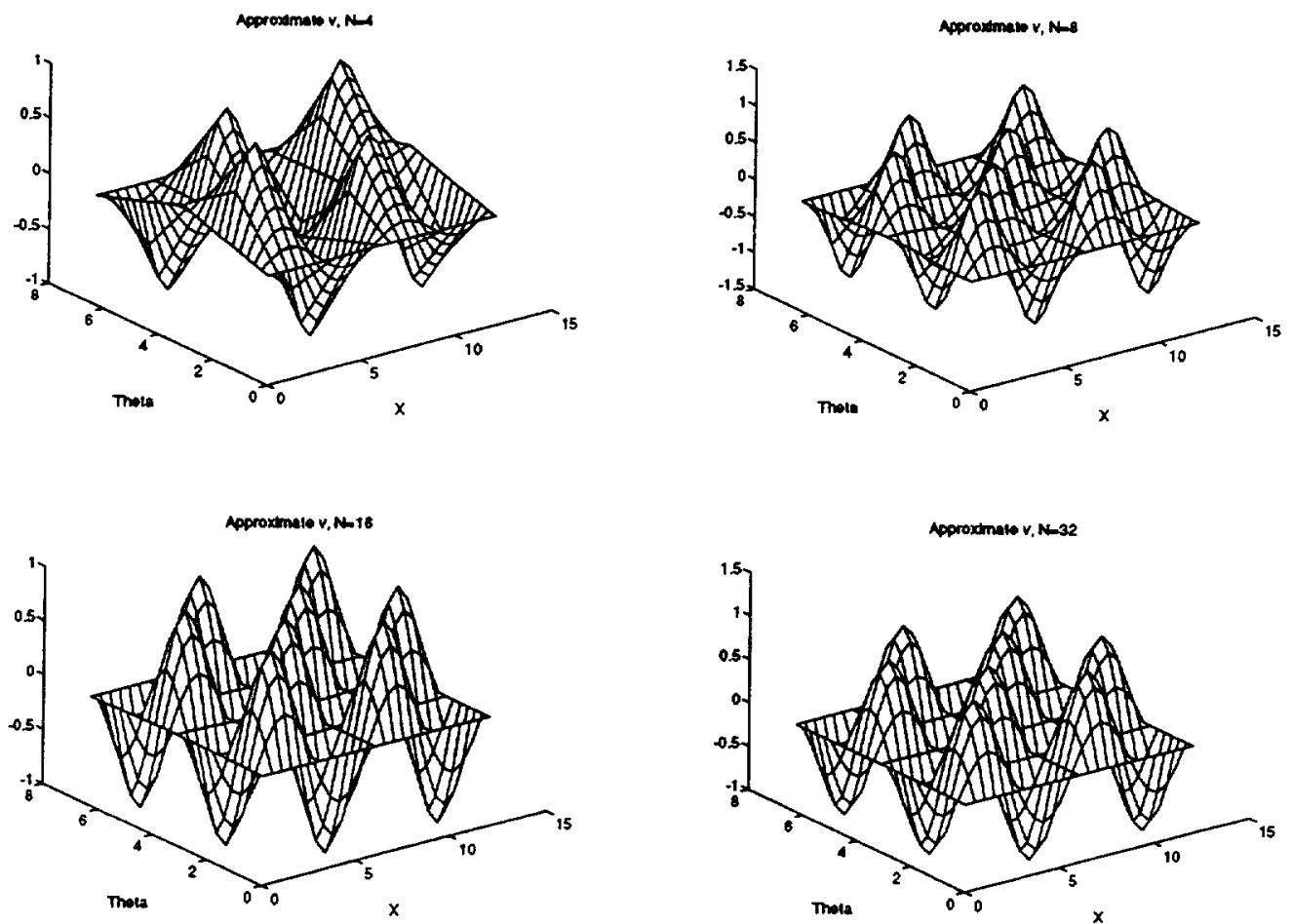

Figure 8. Approximate circumferential displacement $v$ with $N=4,8,16,32$. 

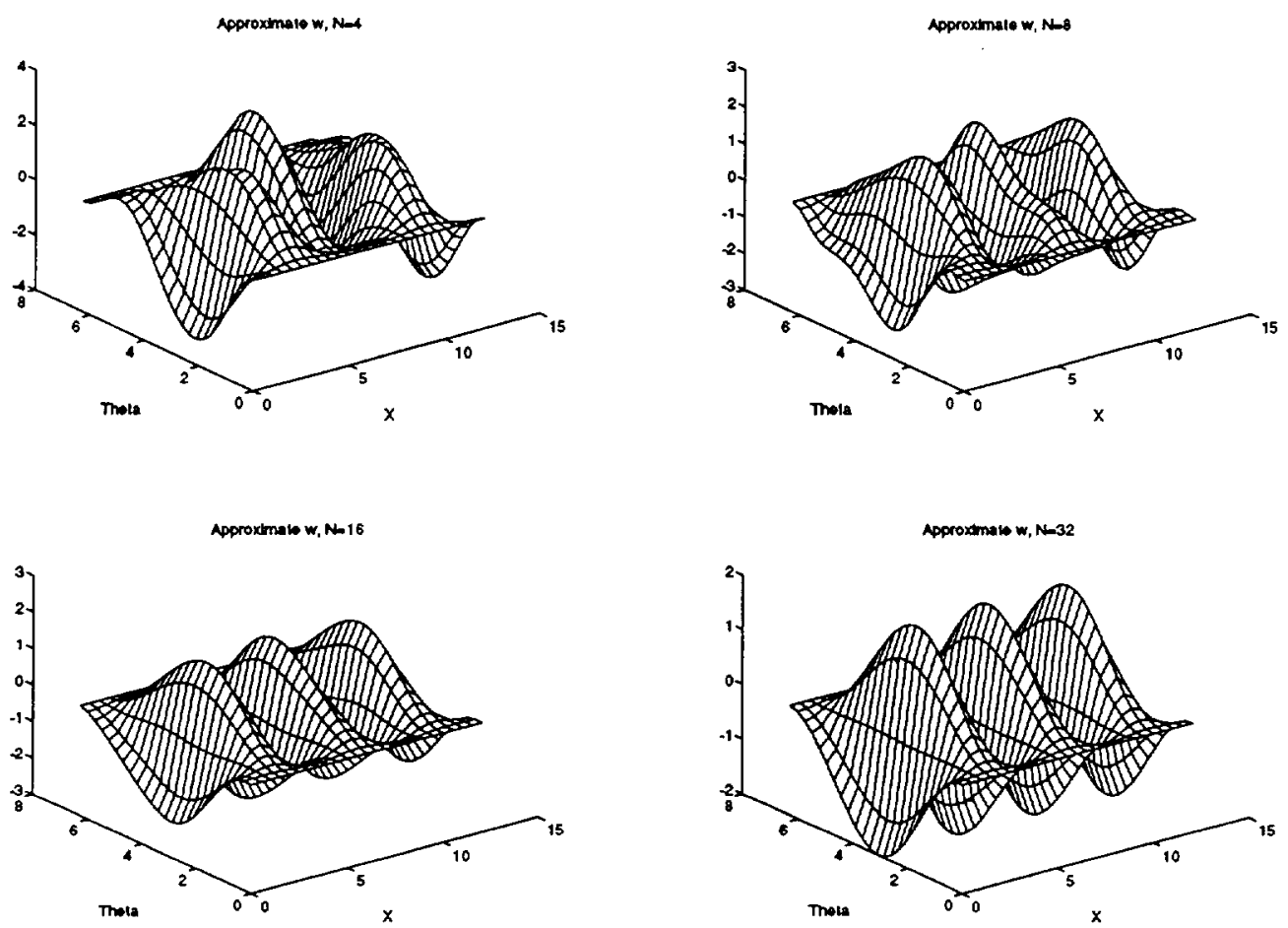

Figure 9. Approximate transverse displacement $w$ with $N=4,8,16,32$.

\subsubsection{Time-Dependent Solution - Cubic Spline Approximation}

To illustrate the approximation of the dynamic shell model (including mass, stiffness, damping and force contributions), time-dependent forcing functions and solutions are considered in the final two examples. Cubic spline axial basis functions are used for discretizing the longitudinal, circumferential and tangential displacements in both cases.

For this example, the true solutions were taken to be

$$
\begin{aligned}
& u(x, \theta)=t^{2} \sin (2 \pi x / \ell) \sin (\theta) \\
& v(x, \theta)=t^{2} \sin (4 \pi x / \ell) \sin (2 \theta) \\
& w(x, \theta)=t^{2}[\cos (2 \pi x / \ell)-1] \cos (3 \theta)
\end{aligned}
$$

(compare with (5.1)). Solving for $\hat{q}_{x}, \hat{q}_{\theta}$ and $\hat{q}_{n}$ in the strong form of the equations (2.1) yields the forcing functions. Since the forcing terms are time-dependent, discretization of the modeling equations yields the matrix equation (4.12) in $R^{N}, N=2 \mathcal{N}$. As detailed in [16], the resulting ODE system is moderately stiff and a variable-stepsize, variable-order stiff ODE routine was used to numerically integrate the system.

The absolute errors in the longitudinal, circumferential and transverse displacements at time $T=1.5 \mathrm{sec}$ are reported in Table 10 while error ratios as $N$ was doubled are reported in Table 11. A comparison between the errors in Table 10 and the steady state errors in Table 8 indicates that they are of the same magnitude while comparison between Tables 11 and 9 
indicates nearly identical error ratios. Both Table 10 and 11 comfirm the $\mathcal{O}\left(h_{x}^{4}\right)$ convergence rate of the method in the damped, time-dependent problem. Hence with the fully cubic spline axial basis, the accuracy contributes to the efficiency of the method through reduced system sizes. This significantly speeds the time marching which is advantageous in simulations, parameter estimation and control applications.

\begin{tabular}{cccc}
\hline & $N=4$ & $N=8$ & $N=16$ \\
\hline$u$ & $3.742-01$ & $4.506-03$ & $8.855-05$ \\
$v$ & $1.280+00$ & $3.376-02$ & $1.403-03$ \\
$w$ & $7.056-01$ & $9.169-03$ & $1.249-04$ \\
\hline
\end{tabular}

Table 10. Absolute errors of the longitudinal $u$, circumferential $v$, and transverse displacements $w$ at time $T=1.5$ seconds.

\begin{tabular}{ccc}
\hline & $N=4$ & $N=8$ \\
\cline { 2 - 3 }$N=8$ & $N=16$ \\
\hline$u$ & 83.052 & 50.888 \\
$v$ & 37.899 & 24.069 \\
$w$ & 76.961 & 73.400 \\
\hline
\end{tabular}

Table 11. Ratios of the absolute errors at time $T=1.5$ seconds.

\subsubsection{Time-Dependent Solution - Patch Input}

In the final example, the external inputs to the patch were assumed to come only from moments and forces generated by a single patch pair located between $x_{1}=4.5 \mathrm{in}, x_{2}=7.5 \mathrm{in}$ and $\theta_{1}=0, \theta_{2}=\pi / 6$. Material and patch parameters are summarized in Table 12. The reported patch parameters are consistent with those of patches currently being used in the Acoustics Division, NASA Langley Research Center.

For this case, the force vector $\hat{F}^{N}(t)$ of $(4.10)$ has the components

$$
\begin{aligned}
{\left[F_{u}(t)\right]_{j} } & =\int_{\theta_{1}}^{\theta_{2}} \int_{x_{1}}^{x_{2}} R\left(N_{x}\right)_{p e}(t) \frac{\partial \mathcal{B}_{u_{j}}}{\partial x} d x d \theta \\
{\left[F_{v}(t)\right]_{j} } & =\int_{\theta_{1}}^{\theta_{2}} \int_{x_{1}}^{x_{2}}\left(N_{\theta}\right)_{p e}(t) \frac{\partial \mathcal{B}_{v_{j}}}{\partial \theta} d x d \theta \\
{\left[F_{w}(t)\right]_{j} } & =-\int_{\theta_{1}}^{\theta_{2}} \int_{x_{1}}^{x_{2}}\left[R\left(M_{x}\right)_{p e}(t) \frac{\partial^{2} \mathcal{B}_{w_{j}}}{\partial x^{2}}+\frac{1}{R}\left(M_{\theta}\right)_{p e}(t) \frac{\partial^{2} \mathcal{B}_{w_{j}}}{\partial \theta^{2}}\right] d x d \theta
\end{aligned}
$$

where $\left(N_{x}\right)_{p e},\left(N_{\theta}\right)_{p e},\left(M_{x}\right)_{p e}$ and $\left(M_{\theta}\right)_{p e}$ are defined in $(2.4)$ with $i=1$. It is noted that due to the characteristic functions, the integrals reduce to only that region covered by the patches. This is in accordance with the physical fact that moments and forces are generated only in patch regions. 
The voltage $V_{1}(t)$ to the outer patch (see (2.5)) was taken to have a triangular shape with a magnitude of $95 \mathrm{~V}$ and a temporal duration of $1 \times 10^{-4}$ seconds. The voltage $V_{2}(t)$ to the inner patch was taken to be $V_{2}(t)=-V_{1}(t)$. This input models an out-of-phase voltage spike to the patches and serves to generate a broadband shell response. The input of such voltages to patches is a current technique for eliciting shell responses when performing system identification or damage detection.

The clamped-edge boundary condition (2.6) was assumed with compatible cubic basis functions in the numerical approximation. The index limits $N=8$ and $M=3$ were sufficient for resolving the shell dynamics in the previous examples, and those limits were also used to obtain the results here. The frequencies and displacements for the point $(x, \theta)=(7.83,0.89)$ are plotted in Figures 10-12 (this point lies just outside the $(x, \theta)=(7.5, \pi / 6)$ corner of the patch). Since out-of-phase simulated voltages were input to the inner and outer patches, the input was primarily in the form of external moments, with only higher order terms contributing to the in-plane forces (see the expressions of (2.5)). The small magnitudes of the displacements results from the use of a single actuating patch pair with a short duration voltage spike. Note that all computations were performed with double precision accuracy.

In Table 13, the natural frequencies obtained through this dynamic patch excitation are compared with those obtained for the undamped shell by solving the matrix eigenvalue problem (4.13). As expected, the two sets of frequencies are nearly identical since the same discretization limits were used in both cases. The slight differences are due to the Kelvin-Voigt damping and sample rates in the dynamic simulation.

\begin{tabular}{|l|l|}
\hline \hline$h=0.01 \mathrm{in}$ & $R=3.00 \mathrm{in}$ \\
$\rho=0.283 \mathrm{lb} / \mathrm{in}^{3}$ & $E=3 \times 10^{7} \mathrm{lb} / \mathrm{in}^{2}$ \\
$\nu=0.3$ & $c_{D}=15.099 \mathrm{lb} \cdot \mathrm{in} \cdot \mathrm{s}$ \\
$\ell=12.0 \mathrm{in}$ & \\
\hline$x_{1}=4.5 \mathrm{in}$ & $x_{2}=7.5 \mathrm{in}$ \\
$\theta_{1}=0$ & $\theta_{2}=\pi / 6$ \\
$E_{p e}=9.137 \times 10^{6} \mathrm{lb} / \mathrm{in}^{2}$ & $\nu_{p e}=0.31$ \\
$h_{p e}=0.001 \mathrm{in}$ & $d_{31}=7.4 \times 10^{-9} \mathrm{in} / \mathrm{V}$ \\
\hline \hline
\end{tabular}

Table 12. Material and piezoceramic patch constants. 


\begin{tabular}{|rr|rr|}
\hline \hline \multicolumn{2}{|c|}{$\begin{array}{c}\text { Dynamically Excited } \\
\text { Frequencies }(\mathrm{Hz})\end{array}$} & \multicolumn{2}{|c|}{ Frequencies from } \\
Eigenvalue Problem (Hz)
\end{tabular}

Table 13. Natural frequencies obtained through dynamic excitation and solution of the matrix eigenvalue problem (4.13).
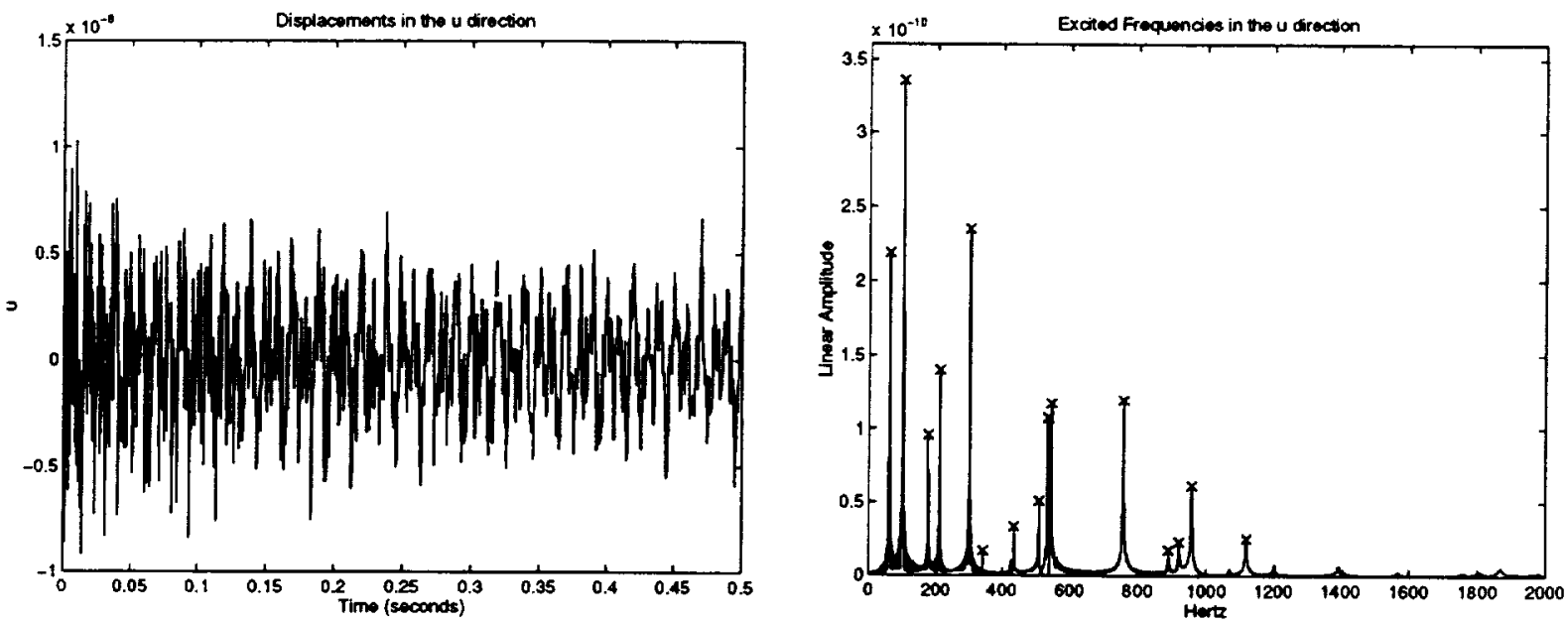

Figure 10. Longitudinal displacements and frequencies for the damped shell.
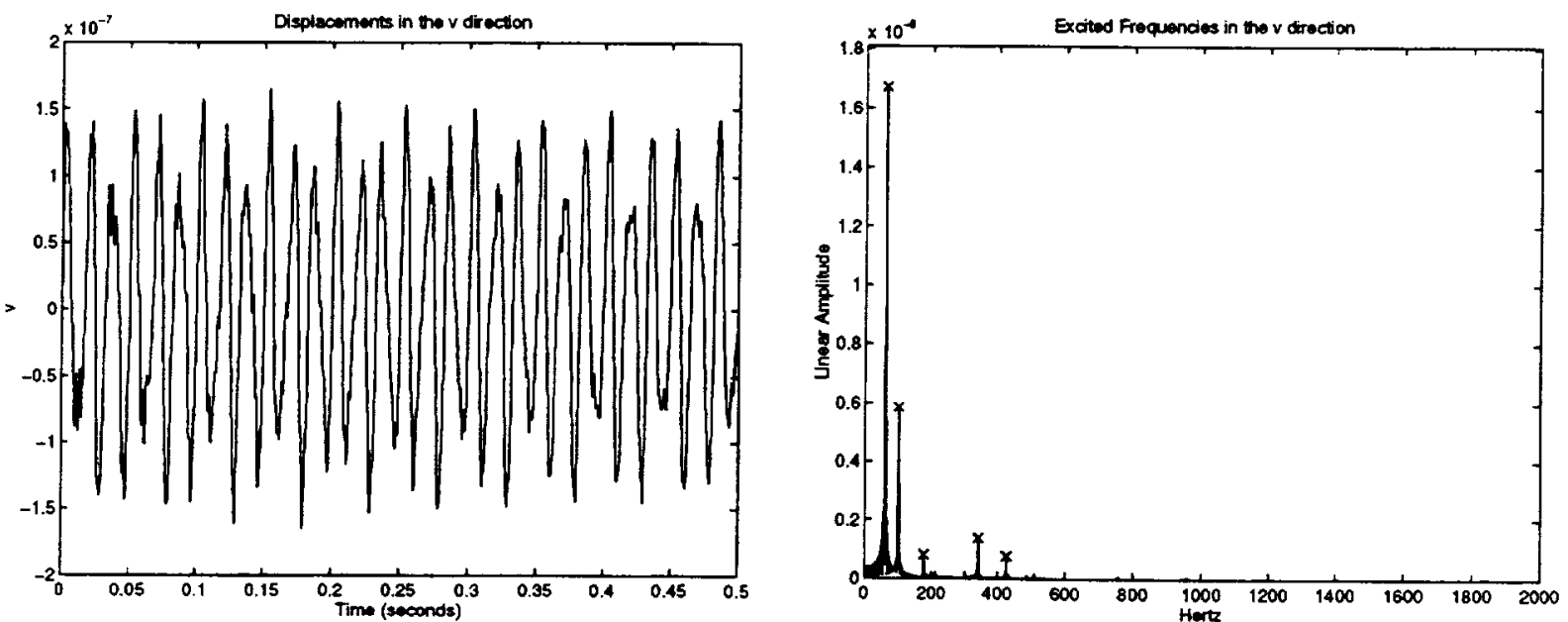

Figure 11. Circumferential displacements and frequencies for the damped shell. 

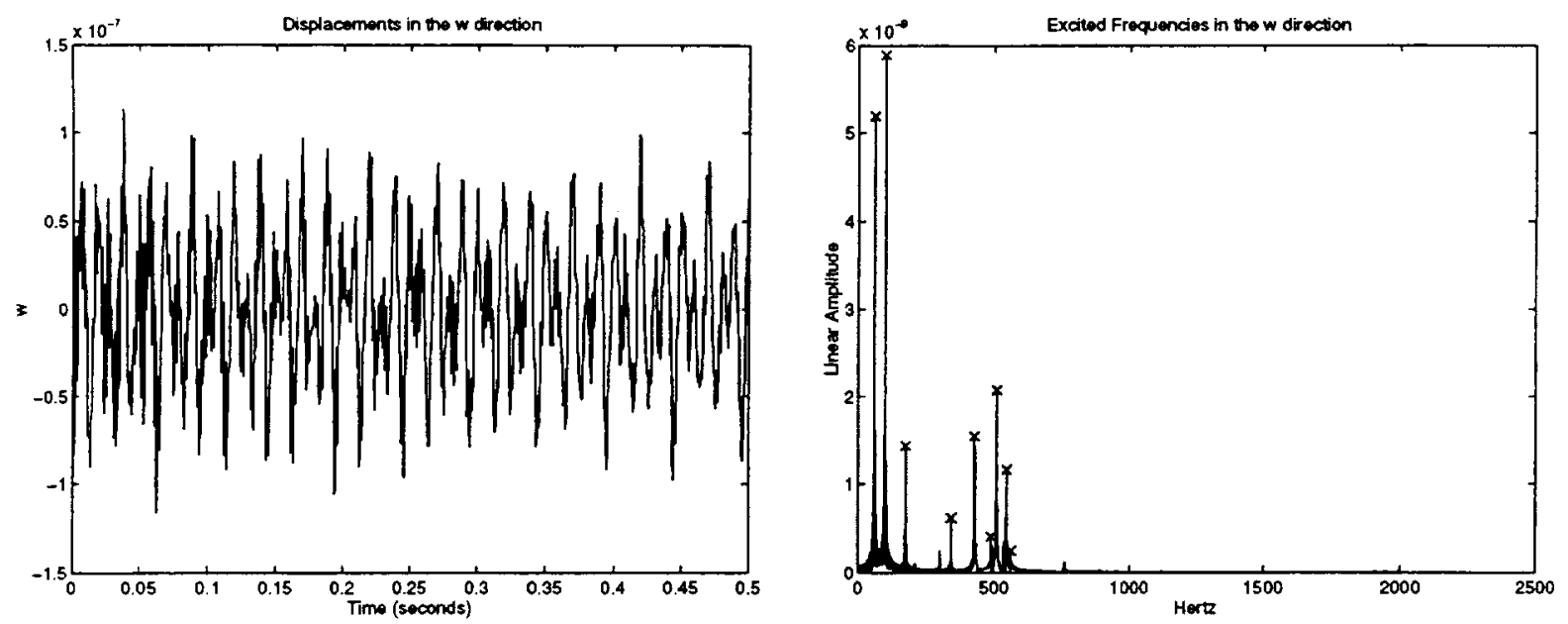

Figure 12. Transverse displacements and frequencies for the damped shell.

\section{Concluding Remarks}

In this paper, a spline-based Galerkin method appropriate for discretizing cylindrical shell models has been presented. The emphasis throughout the discussion was on the development of a method which is sufficiently flexible for a variety of models including those which arise in smart material applications. Galerkin expansions, in terms of basis functions composed of modified splines in the axial direction and Fourier components circumferentially, provided this flexibility. Discretization in this manner also provided an accurate and efficient means of approximating static and dynamic shell deformations as well as natural frequencies and modes for a variety of boundary conditions.

As demonstrated through numerical examples, the method yielded $\mathcal{O}\left(h_{x}^{2}\right)$ convergence for physically realistic values of the shell thickness $h$ when linear splines were used as axial basis elements for $u, v$, and $\mathcal{O}\left(h_{x}^{4}\right)$ accuracy when all three components $u, v, w$ were approximated by cubic spline expansions. The fourth order accuracy attained with cubic splines contributes to the method's efficiency by reducing the number of basis elements and the hence system sizes necessary for resolving the shell behavior (in many of the examples, $N=8$ cubic spline basis functions were sufficient for resolving the displacements $u, v, w)$. Unlike the splines, the Fourier expansions in $\theta$ form a truncated approximate in which all Fourier components with index less than the limit index $M$ are resolved. In applications, the number of excited Fourier modes is often quite small, thus permitting small limits $M$.

Another advantage of the cubic spline basis is the fact that differentiability and smoothness properties are constructed in the basis as compared with many finite element methods in which these conditions are obtained through compatibility conditions at the nodes. This simplifies construction of system matrices since components are constructed simply through the quadrature of basis elements. This also reduces the susceptibility of the method to membrane and shear locking since there are no elements per se in which asymptotic incompatibilities can arise. Alternatively, the spline-based Galerkin method can be thought of as similar to a spectral method but with basis functions having finite support. 
Initial tests have indicated no tendency toward membrane or shear locking when using the fully cubic spline axial bases with small partition size $h_{x}$. This is consistent with finite element results reported in [25]. These locking considerations, in combination with the $\mathcal{O}\left(h_{x}^{4}\right)$ accuracy of the cubic splines, indicate that the fully cubic spline axial basis will be preferable to the mixed linear/cubic basis in most applications.

The method was also demonstrated to be flexible with regards to boundary conditions, and both clamped-end and simply-supported edge conditions were attained through minor modifications in the boundary splines. Free end conditions are natural boundary conditions in the weak model formulation and hence no basis modifications are necessary in that case. "Almost fixed" boundary conditions modeling slight edge rotations and inplane movement are also common in applications due to the difficulty in maintaining perfect boundary clamps. As discussed in [7], only minor modifications are necessary for adopting the splines for this case.

Finally, the approximation method is directly applicable to shell models incorporating passive (internal) and active (external) contributions from embedded or surface-mounted sensors and/or actuators. When derived from physical principles, the passive actuator/sensor contributions are manifested in the physical model parameters (e.g., density, stiffness, damping, Poisson ratio) and are incorporated in the resulting discrete system simply through piecewise integration over the shell domain. The external moments and forces are isolated to regions covered by actuators through characteristic functions in the model. In the weak form, this yields integrals over the actuator regions. While partitions should be aligned with actuator edges to obtain optimal accuracy, neither the basis nor inner products must be modified to account for sensors and/or actuators. This is advantageous when approximating shell dynamics in smart material applications.

\section{Acknowledgements}

The authors express sincere appreciation to I. Babuška, University of Texas at Austin, H.T. Banks, CRSC, North Carolina State University, F.J. Rizzo, Iowa State Univerity and Y. Zhang, CRSC, North Carolina State University, for input regarding various aspects of this investigation.

\section{References}

[1] D.N. Arnold and R.S. Falk, "A Uniformly Accurate Finite Element for the ReissnerMindlin Plate," SIAM Journal of Numerical Analysis, 26, pp. 1276-1290, 1989.

[2] D.G. Ashwell and R.H. Gallagher-ed., Finite Elements for Thin Shells and Curved Members, John Wiley and Sons, New York, 1976.

[3] I. Babuška and M. Suri, "On Locking and Robustness in the Finite Element Method," SIAM Journal of Numerical Analysis, 29(5), pp. 1261-1293, 1992.

[4] I. Babuška and M. Suri, "Locking Effects in the Finite Element Approximation of Elasticity Problems," Numerische Mathematik, 62, pp. 439-463, 1992. 
[5] H.T. Banks and R.C. Smith, "Well-Posedness of a Model for Structural Acoustic Coupling in a Cavity Enclosed by a Thin Cylindrical Shell," Journal of Mathematical Analysis and Applications, 191, pp. 1-25, 1995.

[6] H.T. Banks, R.C. Smith and Y. Wang, "The Modeling of Piezoceramic Patch Interactions with Shells, Plates, and Beams," Quarterly of Applied Mathematics, 53(2), pp. 353-381, 1995.

[7] H.T. Banks, R.C. Smith and Y. Wang, "Modeling and Parameter Estimation for an Imperfectly Clamped Plate," CRSC Technical Report CRSC-TR95-2, North Carolina State Univ., Computation and Control IV, Proc. Fourth Bozeman Conf., Bozeman, MT, 1994, Progress in Systems and Control Theory, Volume 20, Birkhäuser Boston, Inc., pp. 23-42, 1995.

[8] H.T. Banks, R.C. Smith and Y. Wang, "Parameter Estimation for an Imperfectly Clamped Plate - Numerical Examples," Proceedings of the 1995 Design Engineering Technical Conferences, Volume 3, Part C, Boston, MA, September 17-20, pp. 963-972, 1995.

[9] H.T. Banks, R.C. Smith and Y. Wang, Smart Material Structures: Modeling, Estimation and Control, to be published by Masson/Wiley in the collection "Recherches en Mathématiques Appliquées".

[10] K.J. Bathe, F. Brezzi and M. Fortin, "Mixed-Interpolated Elements for ReissnerMindlin Plates," International Journal for Numerical Methods in Engineering, 28, pp. 1787-1801, 1989.

[11] M. Bernardou and J.M. Boisserie, The Finite Element Method in Thin Shell Theory: Applications to Arch Dam Simulations, Birkhäusser, Boston, 1982.

[12] R.D. Blevins, Formulas for Natural Frequency and Mode Shape, Van Nostrand Reinhold Company, New York, 1979.

[13] L.J. Brombolich and P.L. Gould, "Finite Element Analysis of Shells of Revolution by Minimization of the Potential Energy Functional," Proceedings for the Conference on Applications of the Finite Element Method in Civil Engineering, Vanderbilt University, Nashville, TN, November 13-14, 1969.

[14] J.W. Bulle, ed., Finite Element Applications to Thin-Walled Structures, Elsevier Applied Science, New York, 1990.

[15] R.D. Cook, D.S. Malkus and M.E. Plesha, Concepts and Applications of Finite Element Analysis, John Wiley and Sons, New York, 1989.

[16] R.C.H. del Rosario and R.C. Smith, "Spline Approximation of Thin Shell Dynamics - Numerical Examples," Center for Research in Scientific Computation Technical Report CRSC-TR96-5. 
[17] K. Forsberg, "Influence of Boundary Conditions on the Modal Characteristics of Thin Cylindrical Shells," AIAA Journal, 2(12), pp. 2150-2157, 1964.

[18] K. Forsberg, "A Review of Analytical Methods Used to Determine the Modal Characteristics of Cylindrical Shells," NASA CR-613, 1965.

[19] C.R. Fuller, S.D. Snyder, C.H. Hansen and R.J. Silcox, "Active Control of Interior Noise in Model Aircraft Fuselages Using Piezoceramic Actuators," Paper 90-3922, AIAA 13th Aeroacoustics Conference, Tallahassee, FL, October 1990.

[20] P.L. Gould, Finite Element Analysis of Shells of Revolution, Pitman Advanced Publishing Program, Boston, 1985.

[21] P.L. Gould and P.K. Basu, "Geometric Stiffness Matrices for the Finite Element Analysis of Rotational Shells," Journal of Structural Mechanics, 5(1), pp. 87-105, 1977.

[22] H. Harintho and P.L. Gould, "Local-Global Analysis on Localized Non-Linear Shells of Revolutions," Communications in Numerical Methods in Engineering, 10, pp. 933941, 1994.

[23] N.J. Hoff, "The Accuracy of Donnell's Equations," Journal of Applied Mechanics, 22, pp. 329-334, 1955.

[24] H. Kraus, Thin Elastic Shells: An Introduction to the Theoretical Foundations and the Analysis of Their Static and Dynamic Behavior, John Wiley and Sons, Inc., New York, 1967.

[25] Y. Leino and J. Pitkäranta, "On the Membrane Locking of $h-p$ Finite Elements in a Cylindrical Shell Problem," International Journal for Numerical Methods in Engineering, 37, pp. 1053-1070, 1994.

[26] A.W. Leissa, Vibration of Shells, NASA SP-288, 1973; Reprinted by the Acoustical Society of America through the American Institute of Physics, 1993.

[27] S. Markuš, The Mechanics of Vibrations of Cylindrical Shells, Elsevier, New York, 1988.

[28] J. Pitkäranta, "The Problem of Membrane Locking in Finite Element Analysis of Cylindrical Shells," Numerische Mathematik, 61, pp. 523-542, 1992.

[29] P.M. Prenter, Splines and Variational Methods, Wiley Classics Edition, New York, 1989.

[30] G. Rengarajan, M.A. Aminpour and N.F. Knight, Jr., "Improved Assumed-Stress Hybrid Shell Element with Drilling Degrees of Freedom for Linear Stress, Buckling and Free Vibration Analyses," International Journal for Numerical Methods in Engineering, 38, pp. 1917-1943, 1995. 
[31] M. Soare, Application of Finite Difference Equations to Shell Analysis, Pergamon Press, New York, 1967.

[32] W. Soedel, Vibrations of Shells and Plates, 2nd ed., Marcel Dekker, New York, 1993.

[33] W. Soedel and M. Dhar, "Difficulties in Finding Modes Experimentally when Several Contribute to a Resonance," Journal of Sound and Vibration, 58(1), pp. 27-38, 1978.

[34] H.S. Tzou, Piezoelectric Shells: Distributed Sensing and Control of Continua, Kluwer Academic Publishers, Boston, 1993. 


\begin{tabular}{|c|c|c|c|}
\hline \multicolumn{3}{|c|}{ REPORT DOCUMENTATION PAGE } & $\begin{array}{l}\text { Form Approved } \\
\text { OMB No. } 0704-0188\end{array}$ \\
\hline \multicolumn{4}{|c|}{ 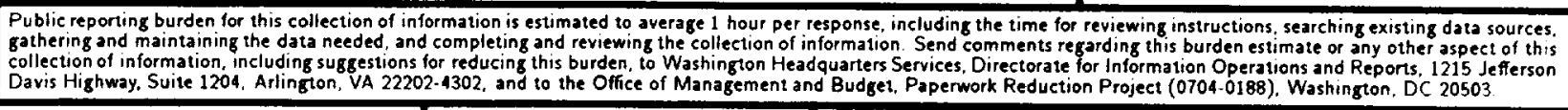 } \\
\hline 1. AGENCY USE ONLY(Leave blank) & $\begin{array}{l}\text { 2. REPORT DATE } \\
\text { March } 1996\end{array}$ & \multicolumn{2}{|c|}{$\begin{array}{l}\text { 3. REPORT TYPE AND DATES COVERED } \\
\text { Contractor Report }\end{array}$} \\
\hline \multicolumn{3}{|c|}{$\begin{array}{l}\text { 4. TITLE AND SUBTITLE } \\
\text { SPLINE APPROXIMATION OF THIN SHELL DYNAMICS }\end{array}$} & \multirow[t]{2}{*}{$\begin{array}{l}\text { 5. FUNDING NUMBERS } \\
\text { C NAS1-19480 } \\
\text { WU } 505-90-52-01\end{array}$} \\
\hline \multicolumn{3}{|l|}{$\begin{array}{l}\text { 6. AUTHOR(S) } \\
\text { R. C. H. del Rosario } \\
\text { R. C. Smith }\end{array}$} & \\
\hline \multicolumn{3}{|c|}{$\begin{array}{l}\text { 7. PERFORMING ORGANIZATION NAME(S) AND ADDRESS(ES) } \\
\text { Institute for Computer Applications in Science and Engineering } \\
\text { Mail Stop 132C, NASA Langley Research Center } \\
\text { Hampton, VA } 23681-0001\end{array}$} & $\begin{array}{l}\text { 8. PERFORMING ORGANIZATION } \\
\text { REPORT NUMBER } \\
\text { ICASE Report No. } 96-26\end{array}$ \\
\hline \multicolumn{3}{|c|}{$\begin{array}{l}\text { 9. SPONSORING/MONITORING AGENCY NAME(S) AND AODRESS(ES) } \\
\text { National Aeronautics and Space Administration } \\
\text { Langley Research Center } \\
\text { Hampton, VA 23681-0001 }\end{array}$} & $\begin{array}{l}\text { 10. SPONSORING/MONITORING } \\
\text { AGENCY REPORT NUMBER } \\
\text { NASA CR-198323 } \\
\text { ICASE Report No. } 96-26\end{array}$ \\
\hline \multicolumn{4}{|c|}{$\begin{array}{l}\text { 11. SUPPLEMENTARY NOTES } \\
\text { Langley Technical Monitor: Dennis M. Bushnell } \\
\text { Final Report } \\
\text { Submitted to International Journal for Numerical Methods in Engineering. }\end{array}$} \\
\hline \multicolumn{2}{|c|}{$\begin{array}{l}\text { 12a. DISTRIBUTION/AVAILABILITY STATEMENT } \\
\text { Unclassified-Unlimited } \\
\text { Subject Category } 64\end{array}$} & 12b. DIST & 12b. DISTRIBUTION CODE \\
\hline \multicolumn{4}{|c|}{$\begin{array}{l}\text { 13. ABSTRACT (Maximum } 200 \text { mords) } \\
\text { A spline-based method for approximating thin shell dynamics is presented here. While the method is developed } \\
\text { in the context of the Donnell-Mushtari thin shell equations, it can be easily extended to the Byrne-Flügge-Lur'ye } \\
\text { equations or other models for shells of revolution as warranted by applications. The primary requirements for the } \\
\text { method include accuracy, flexibility and efficiency in smart material applications. To accomplish this, the method } \\
\text { was designed to be flexible with regard to boundary conditions, material nonhomogeneities due to sensors and } \\
\text { actuators, and inputs from smart material actuators such as piezoceramic patches. The accuracy of the method was } \\
\text { also of primary concern, both to guarantee full resolution of structural dynamics and to facilitate the development } \\
\text { of PDE-based controllers which ultimately require real-time implementation. Several numerical examples provide } \\
\text { initial evidence demonstrating the efficacy of the method. }\end{array}$} \\
\hline \multirow{2}{*}{\multicolumn{2}{|c|}{$\begin{array}{l}\text { 14. SUBJECT TERMS } \\
\text { Galerkin Method; Thin Shell Dynamics }\end{array}$}} & 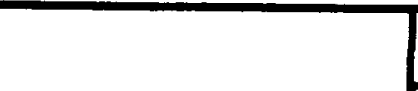 & $\begin{array}{l}\text { 15. NUMBER OF PAGES } \\
39\end{array}$ \\
\hline & & & $\begin{array}{c}\text { 16. PRICE CODE } \\
\mathrm{A} 03\end{array}$ \\
\hline $\begin{array}{l}\text { 17. SECURITY CLASSIFICATION } \\
\text { OF REPORT } \\
\text { Unclassified }\end{array}$ & $\begin{array}{l}\text { 18. SECURITY CLASSIFICATION } \\
\text { OF THIS PAGE } \\
\text { Unclassified }\end{array}$ & $\begin{array}{l}\text { 19. SECURITY CLASSIFICATION } \\
\text { OF ABSTRACT }\end{array}$ & $\begin{array}{l}\text { 20. LIMITATION } \\
\text { OF ABSTRACT }\end{array}$ \\
\hline SN 7540-01-280-5500 & & & $\begin{array}{l}\text { Standard Form } 298(\text { Rev. 2-89) } \\
\text { Prescribed by ANSI Std. 239-18 } \\
\text { 298-102 }\end{array}$ \\
\hline
\end{tabular}


